\title{
LE NORD-PAS-DE-CALAIS, ENTRE MUTATIONS ÉCONOMIQUES ET DÉVELOPPEMENT HUMAIN
}

\author{
Grégory Marlier, Conseil régional du Nord-Pas-de-Calais (D2DPE) \\ Thomas Dallery, Université du Littoral Côte d'Opale \\ Nathalie Chusseau, Université de Lille 1
}

La région Nord-Pas-de-Calais connaît depuis plusieurs décennies une transformation de son tissu productif qui pose la question de l'arbitrage entre mutation économique et progrès social : là où les difficultés économiques ont été les plus marquées, des territoires ont pu connaître un réel décrochage, avec de moindres perspectives de progrès social. Cette problématique est accentuée depuis 2008, avec une crise qui a des conséquences importantes sur les territoires.

Cet article comporte trois parties. La première aborde les différentes mutations de l'économie du Nord-Pas-de-Calais, une économie qui connaît une progression plus favorable que la moyenne nationale, aussi bien en période de croissance qu'en période de crise. La construction d'une maquette de la macroéconomie régionale permet de mettre en évidence une certaine autonomie que les décideurs publics peuvent exploiter. La deuxième partie présente les différents moteurs du développement local pour aboutir à une typologie des profils des territoires de la région Nord-Pas-de-Calais, au regard des mutations et polarités économiques. Enfin, la troisième partie apporte un autre regard sur le développement régional à partir d'indicateurs alternatifs d'inégalités territoriales soulignant une possible contradiction entre développement économique d'un côté et développement humain et durable de l'autre.

Mots clés : mutations et polarités économiques, inégalités territoriales, développement humain, santé sociale, multiplicateur keynésien, théorie de la base.

\section{L} a seconde moitié $\mathrm{du} \mathrm{xx}^{\mathrm{e}}$ siècle a connu une transformation radicale de l'économie française avec un passage de l'économie industrielle à l'économie tertiaire qui a déstabilisé les territoires les plus industrialisés, en particulier celui de la région Nord-Pas- de-Calais. 
Les effets conjugués de la mutation économique et des gains de productivité ont affecté les nombreux espaces nordistes monoindustriels. Dans les zones touchées, la faiblesse des qualifications des salariés et leur manque de diversification sont autant d'obstacles à l'attractivité de ces territoires. La région Nord-Pas-de-Calais connaît depuis plusieurs décennies une transformation de son tissu productif qui pose la question de l'arbitrage entre mutation économique et progrès social : là où les difficultés économiques ont été les plus marquées, des territoires ont pu connaître un réel décrochage, avec de moindres perspectives de progrès social. Cette problématique est accentuée depuis 2008, avec une crise qui a, et aura encore davantage à l'avenir, des conséquences importantes sur les territoires. Les différentes dimensions de cette crise réclament d'autant plus de capacités collectives de compréhension, d'interprétation et d'actions.

La mesure des inégalités multidimensionnelles entre les différentes composantes des territoires permet précisément de fixer le cap de l'action publique régionale et territoriale : de la performance globale des territoires et de leur résilience face aux mutations en cours dépendront les conditions dans lesquelles s'opéreront les régulations à mettre en place.

Dans ce contexte, le niveau régional est un des pivots de ces régulations, ce dernier pouvant permettre l'expression d'un intérêt commun capable de rassembler et permettant aussi l'expression de la spécificité des différentes échelles territoriales fondée sur une inter-territorialité progressive et volontaire.

Cette contribution s'appuie sur trois parties. La première aborde les différentes mutations de l'économie du Nord-Pas-de-Calais, une économie qui connaît une progression plus favorable qu'en moyenne nationale, aussi bien en période de croissance qu'en période de crise. La deuxième partie présente les différents moteurs du développement local pour aboutir à une typologie des profils économiques des territoires de la région Nord-Pas-de-Calais. Enfin, la troisième partie présentera un autre regard sur le développement régional à partir d'indicateurs d'inégalités territoriales mettant en évidence une possible contradiction entre développement économique d'un côté et développement humain et durable de l'autre. 


\section{Une région en pleine mutation économique}

\subsection{Situation du Nord-Pas-de-Calais dans I'espace économique national et dans la crise}

En 2013, la production mesurée par le PIB représente un peu plus de 106 milliards d'euros plaçant la région en quatrième position parmi les régions de France métropolitaine, ce qui correspond à son classement selon le poids démographique des régions. Toutefois, le PIB régional représente $5,1 \%$ du PIB de France métropolitaine alors que la région représente $6,4 \%$ de la population. Cet écart est en partie lié à l'importance de l'Île-de-France qui produit plus de $30 \%$ de la richesse nationale pour $19 \%$ de la population. Toutefois, la production régionale par habitant reste inférieure à la moyenne de la France de province.

En rapportant la production au nombre d'habitants de la région, le PIB par habitant du Nord-Pas-de-Calais s'élève à 26137 euros en 2013 contre 32317 euros par habitant en France métropolitaine et 27698 euros par habitant en province française (tableau 1). Le PIB par habitant régional est ainsi de $20 \%$ inférieur au PIB par habitant de France métropolitaine et de $6 \%$ inférieur au PIB par habitant de France de province. Le Nord-Pas-de-Calais se place finalement en $16^{\mathrm{e}}$ position parmi les régions de France métropolitaine, loin derrière de grandes régions comme RhôneAlpes (32 219 euros par habitant) ou Provence-Alpes-Côte-d'Azur (30 800 euros par habitant).

Dans la mesure du PIB par habitant, la région Nord-Pas-deCalais est pénalisée par des taux d'activité en retrait par rapport aux autres régions métropolitaines et par un taux de chômage élevé.

En rapportant la production au nombre d'emplois présents sur le territoire, le PIB par emploi du Nord-Pas-de-Calais représente 99 \% de la valeur moyenne de la France de province. En 2013, le PIB par emploi s'élève finalement à 70307 euros contre 71139 euros en moyenne en France de province. En dehors de l'Île-de-France, seules deux régions ont un niveau nettement supérieur à cette valeur moyenne: Rhône-Alpes et Provence-AlpesCôte-d'Azur. La région se positionne ainsi en $9^{\mathrm{e}}$ position au regard du PIB par emploi. 
Tableau 1. PIB par habitant et par emploi des régions françaises

\begin{tabular}{|c|c|c|c|c|}
\hline & \multicolumn{2}{|c|}{ PIB par habitant } & \multicolumn{2}{|c|}{ PIB par emploi } \\
\hline & 2012 & $\begin{array}{c}2013 \\
\text { (provisoire) }\end{array}$ & 2012 & $\begin{array}{c}2013 \\
\text { (provisoire) }\end{array}$ \\
\hline Alsace & 29528 & 29767 & 71771 & 72777 \\
\hline Aquitaine & 28323 & 28279 & 70489 & 70514 \\
\hline Auvergne & 25945 & 26260 & 66696 & 67526 \\
\hline Bourgogne & 26727 & 26832 & 67529 & 68226 \\
\hline Bretagne & 26655 & 26630 & 66400 & 66796 \\
\hline Centre & 26847 & 26803 & 68870 & 69271 \\
\hline Champagne-Ardenne & 27833 & 28355 & 70667 & 72796 \\
\hline Corse & 26554 & 26887 & 68716 & 69787 \\
\hline Franche-Comté & 24429 & 24547 & 64747 & 65800 \\
\hline île-de-France & 52298 & 52729 & 103256 & 104149 \\
\hline Languedoc-Roussillon & 24433 & 24556 & 69204 & 70251 \\
\hline Limousin & 23839 & 24061 & 62432 & 63197 \\
\hline Lorraine & 24097 & 24190 & 67433 & 68524 \\
\hline Midi-Pyrénées & 28627 & 28657 & 69795 & 69919 \\
\hline Nord-Pas-de-Calais & 25822 & 26137 & 69135 & 70307 \\
\hline Basse-Normandie & 26157 & 26324 & 67003 & 67868 \\
\hline Haute-Normandie & 28027 & 27864 & 73068 & 73371 \\
\hline Pays de la Loire & 28646 & 28773 & 69437 & 70183 \\
\hline Picardie & 24450 & 24637 & 70640 & 71725 \\
\hline Poitou-Charentes & 26058 & 25995 & 67354 & 67544 \\
\hline Provence-Alpes-Côte d'Azur & 30337 & 30800 & 75190 & 76553 \\
\hline Rhône-Alpes & 32010 & 32219 & 75447 & 76365 \\
\hline Province & 27698 & 27856 & 70362 & 71169 \\
\hline Métropole & 32317 & 32527 & 77903 & 78762 \\
\hline France entière & 31878 & 32084 & 77615 & - \\
\hline
\end{tabular}

Source : Insee base 2010.

Entre 1990 et 2008, la croissance globale de la production mesurée par le PIB est plus faible qu'au niveau national (graphique 1). Les moindres performances du PIB sont à mettre en relation avec la moindre augmentation de la population: le rapport de la DIACT réalisé en janvier 2009 montre le lien entre le solde migratoire et la croissance du PIB au sein des régions (graphique 2). Ainsi, les régions du Sud et du littoral atlantique apparaissent doublement favorisées avec une croissance du PIB plus élevée et par une croissance de la population liée à l'attracti- 
vité résidentielle. À l'inverse, les régions du quart Nord-Est, dont le Nord-Pas-de-Calais, sont doublement pénalisées par une progression plus faible du PIB et par une faible évolution de la population liée au déficit migratoire. Le Nord-Pas-de-Calais semble toutefois dans une position plus favorable au cours des dernières années de cette période en étant la seule région de la zone Nord-Est à connaître une croissance du PIB plus élevée qu'en moyenne nationale entre 2004 et 2008.

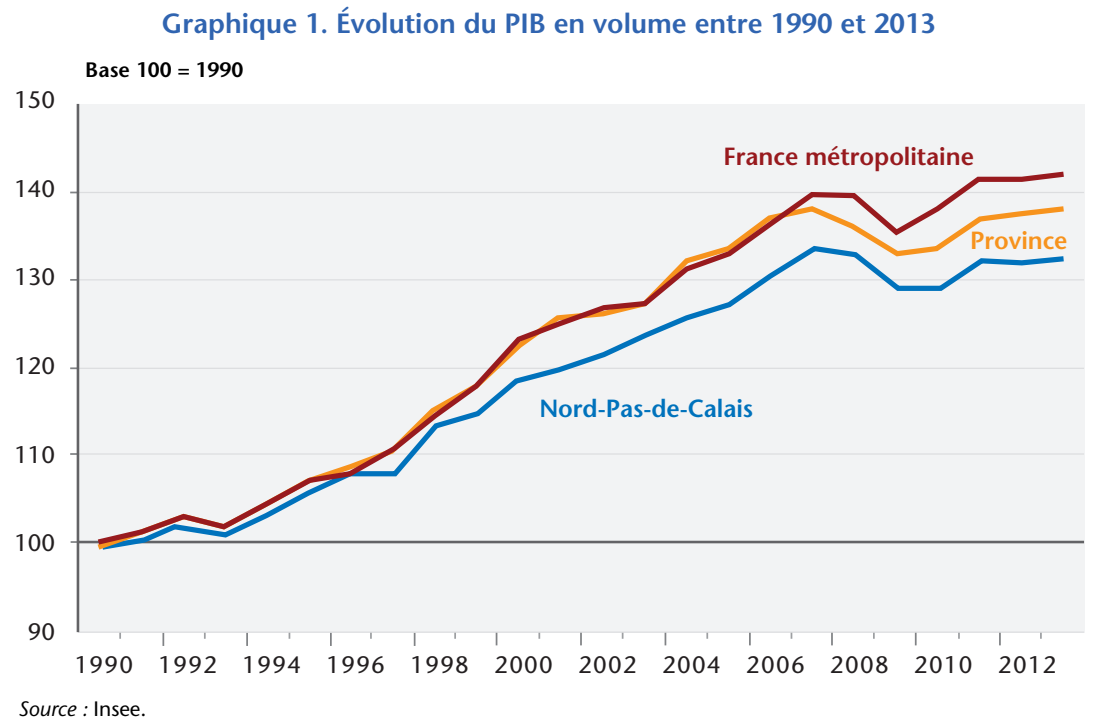

Le PIB par habitant a progressé plus rapidement dans le NordPas-de-Calais qu'en France métropolitaine et en France de province : la région Nord-Pas-de-Calais est la $4^{\mathrm{e}}$ du pays ayant eu la plus forte hausse du PIB par habitant au cours de cette période derrière la Bretagne, les Pays-de-la-Loire et la Corse. Le rattrapage est le plus important entre 2001 et 2008 avec la plus importante hausse du PIB par habitant en évolution relative et la troisième plus forte hausse en euros par habitant derrière l'île-de-France et Provence-Alpes-Côte-d'Azur.

À prix courant, le PIB du Nord-Pas-de-Calais de 2013 représente 99,4\% du volume du PIB de l'année 2007. La région a ainsi retrouvé son niveau de production économique d'avant-crise, même si ce retour s'est traduit par d'importantes restructurations. 
À l'échelle nationale, ce retour au niveau global d'avant-crise s'est produit plus tôt en raison d'un comportement différent de l'Île-deFrance et d'une croissance globalement plus importante de la région capitale. L'évolution du Nord-Pas-de-Calais se rapproche par contre de l'évolution moyenne de la France de province, même si les évolutions ont été très contrastées entre régions au cours de cette période. Ces variations du PIB proches de la moyenne nationale dans un contexte de stabilité démographique se traduisent par une meilleure performance du PIB par habitant qui a progressé de façon nettement plus favorable que la moyenne de France de province, y compris au cours de cette période.

Le lien entre l'évolution du volume de production de richesse et la croissance de la population observée dans d'autres régions en période de croissance s'observe également sur la période 20072013. Pour le Nord-Pas-de-Calais, l'évolution du PIB a été meilleure qu'attendue compte tenu de la faible croissance démographique. Cet écart confirme ainsi un certain dynamisme économique observé sur la période 2004-2008. En dehors de l'Île-de-France, le Nord-Pas-de-Calais est d'ailleurs la seule région à avoir une évolution du PIB nettement supérieure à celle attendue, aussi bien avant la crise que pendant la crise. Cette situation tranche avec les régions voisines plus touchées par les impacts de la crise au regard de l'évolution du PIB entre 2007 et 2013 (graphique 2b).

Malgré ce rattrapage récent, il est néanmoins à mettre au débit de la région un retard dans l'entreprenariat avec une faible propension à la création d'entreprises : la densité d'établissements est particulièrement faible lorsqu'on la rapporte à son nombre d'habitants; pour 1000 habitants dans le Nord-Pas-de-Calais, 32,3 établissements sont présents contre 48,4 en moyenne nationale. Toujours du côté des points noirs, la région souffre d'un net retard dans la Recherche \& développement, et plus globalement une certaine contre-performance dans le domaine de l'économie de la connaissance, puisque le taux de diplômés du supérieur y est aussi plus faible qu'en moyenne nationale. La région Nord-Pas-de-Calais n'a d'ailleurs pas encore atteint l'objectif de $40 \%$ de diplômés du supérieur parmi les 30-34 ans fixé dans le cadre de la stratégie Europe 2020 alors que la France a déjà atteint cet objectif. 
Graphique 2. Évolution du PIB et de la population

a) Entre 1990 et 2007

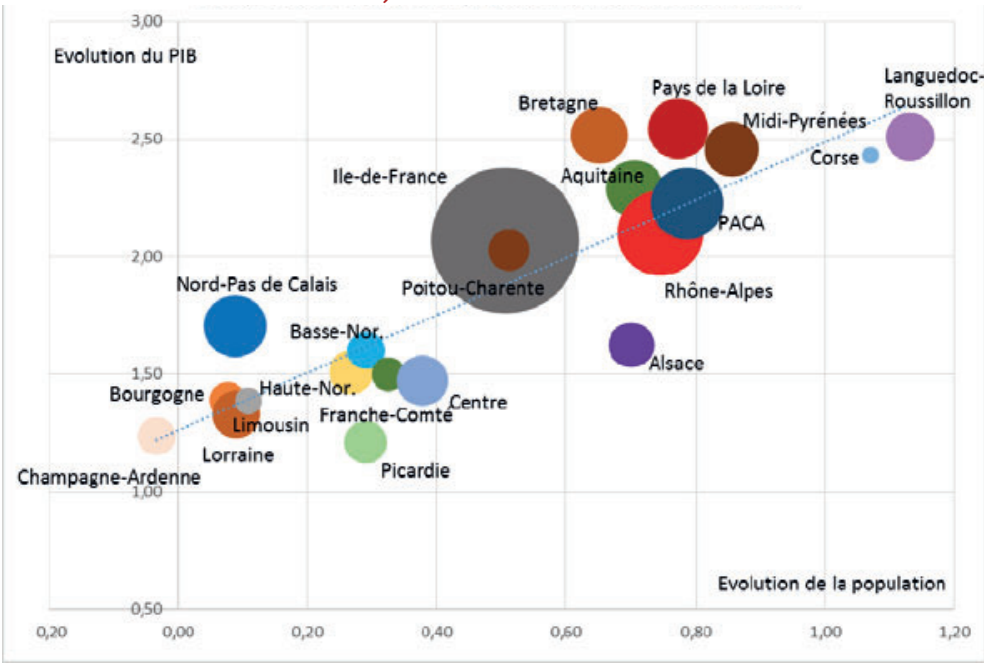

b) Entre 2007 et 2013

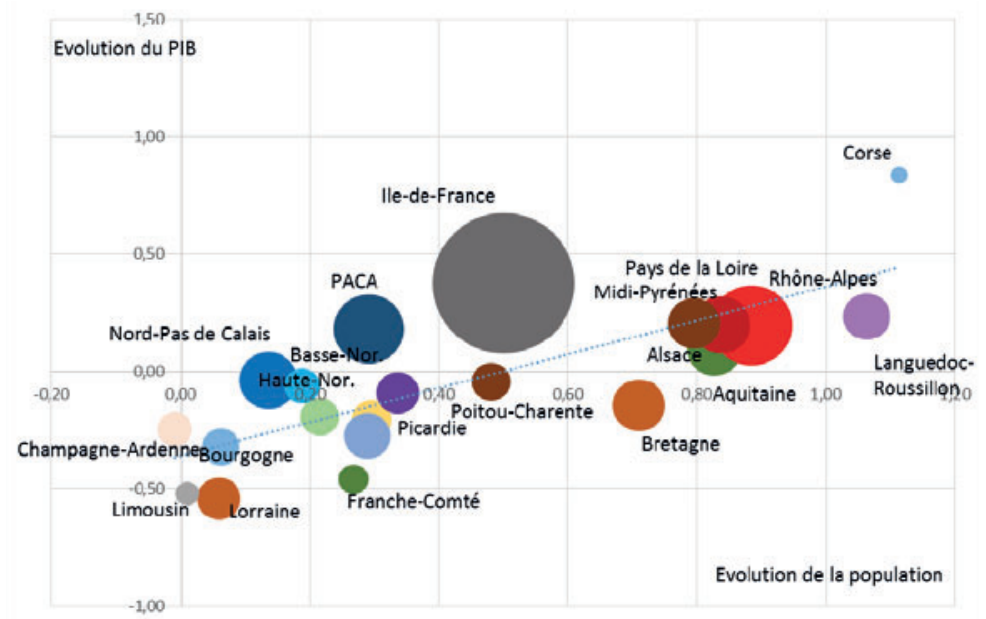

Source : Insee.

\subsection{Une sphère productive reconvertie}

Au cours de ces vingt dernières années, le Nord-Pas-de-Calais a connu d'importantes mutations de la structure productive de son économie (INSEE Nord-Pas-de-Calais, 2014).

En 1990, malgré les restructurations à l'œuvre depuis les années 1970, l'industrie reste le principal secteur d'activité avec 27 \% de la 
valeur ajoutée régionale. Entre 1990 et 2013, la part de l'industrie dans la valeur ajoutée est divisée presque par deux. Le poids de l'industrie dans l'économie régionale représente aujourd'hui celui observé en France de province (15\%). Au cours de la crise récente, les baisses d'emplois dans les secteurs de l'industrie se sont confirmées puisque les secteurs industriels apparaissent comme les secteurs les plus touchés par les impacts de la crise. Dans le même temps, la part des services marchands est en croissance continue, passant de $24 \%$ au début des années 1990 à un peu plus de $32 \%$ vingt ans plus tard. Cette tertiarisation provient notamment de l'essor des services aux entreprises comme les activités spécialisées, scientifiques et techniques, les activités de services administratifs et de soutien, dont le poids dans la valeur ajoutée régionale passe de $8 \%$ à $11 \%$. Cette évolution traduit à la fois le développement de l'économie de la connaissance mais aussi un mouvement d'externalisation des services par les entreprises industrielles. Par ailleurs, depuis 1990, la part des activités immobilières s'est accrue de quatre points et atteint aujourd'hui $12 \%$. Les services non marchands, qui relèvent pour l'essentiel de la sphère publique, sont particulièrement présents dans la région : ils représentent environ $27 \%$ de la valeur ajoutée totale aujourd'hui, contre $25 \%$ en France de province. Ils se sont davantage développés au début des années 1990 et à la suite de la récession de 2008-2009. La part des autres secteurs (commerce, hébergement, restauration, construction, agriculture) dans la valeur ajoutée régionale est restée relativement stable. Entre 1990 et 2011, les sources de la croissance régionale sont assez différentes de celles observées dans les autres régions métropolitaines. Le tertiaire marchand explique près des trois quarts de la croissance de la valeur ajoutée : $53 \%$ pour les services marchands et $20 \%$ pour le commerce, le transport et l'hébergement-restauration. En France de province, sa contribution est inférieure de dix points, en raison notamment d'une moindre dynamique des services marchands. Autre spécificité régionale, l'industrie ne contribue pas à la croissance de la valeur ajoutée, en raison notamment de la forte baisse de la valeur ajoutée industrielle entre 2008 et 2011. En France de province, l'industrie explique $15 \%$ de la croissance. Ce contraste s'explique notamment par une moindre hausse de la productivité dans l'industrie $(+2,1 \%$ par an dans la région, contre $+2,7 \%$ en France de province) et un 
recul plus prononcé de l'emploi dans la région. La valeur ajoutée industrielle a ainsi stagné en Nord-Pas-de-Calais alors qu'elle progressait de 1,3\% par an en France de province. Enfin, avec $25 \%$, la contribution du tertiaire non-marchand à la croissance est significative. Les autres secteurs d'activité (agriculture et construction) contribuent de façon marginale à la croissance régionale.

La région se caractérise également par des spécialisations très marquées et reconnues par la labellisation de 7 pôles de compétitivité. Les entreprises adhérentes à ces pôles emploient près de 50000 personnes dans la région.

Ainsi, la vente à distance représente près de 12000 emplois dans la région fin 2008, soit $46 \%$ des effectifs nationaux du secteur. La région bénéficie plus généralement d'un savoir-faire dans le secteur du commerce avec une première place dans le secteur de la grande distribution grâce en particulier à la présence du groupe Auchan France. Ce savoir-faire s'articule autour du pôle « industrie du commerce ».

L'industrie ferroviaire est également une spécialisation régionale avec la présence sur son territoire des deux principaux acteurs du secteur: Alstom et Bombardier. Avec plus de 4000 emplois dans ce secteur, la région concentre plus de $36 \%$ des emplois salariés du secteur. Ce secteur constitue la clé de voûte du pôle " i-trans ",seul pôle de compétitivité à vocation mondiale du NordPas-de-Calais. Ce pôle associe également plusieurs entreprises de la logistique, de l'automobile et de la métallurgie. La région est en particulier la première région employeuse dans le secteur de la métallurgie-sidérurgie et deuxième région employeuse dans l'industrie automobile.

Le Nord-Pas-de-Calais est également la première région exportatrice pour l'industrie alimentaire. Cette industrie qui s'appuie sur de grands leaders européens du secteur emploie ainsi plus 27300 salariés fin 2008. L'industrie agro-alimentaire est à la fois représentée dans le pôle «NSL, Nutrition Longévité, Santé »- où plusieurs acteurs de la filière sont associés aux sociétés de biotechnologies innovantes et aux laboratoires de recherche - et dans le pôle "MAUD, Matériaux et Applications pour une Utilisation Durable ", dans lequel Roquette constitue, avec Arc International, le principal établissement privé de la région. Le pôle associe égale- 
ment plusieurs entreprises régionales du papier-carton et des industries graphiques, secteurs dans lesquels la région se place au troisième rang au niveau national.

Si l'industrie textile a connu un déclin encore plus marqué dans le Nord-Pas-de-Calais que dans le reste de la France métropolitaine, le secteur régional a également su s'adapter par le développement des textiles techniques permettant au Nord-Pas-de-Calais d'être la deuxième région industrielle dans ce secteur innovant qui s'organise autour du pôle «Up-tex ». Organisé autour de la filière aquatique, le pôle de compétitivité «Aquimer » regroupe à la fois professionnels de la pêche, entreprises agroalimentaires, laboratoires d'étude et de recherche, centres techniques et organismes de formation.

Dernier pôle labellisé, TEAM $^{2}$ regroupe 122 entreprises du secteur de la gestion des déchets et des utilisateurs de matières premières secondaires. Ce pôle apparaît comme un atout régional pour les enjeux de développement durable et la création d'emplois de l'« économie verte ».

La région Nord-Pas-de-Calais reste également marquée par l'importance des grands groupes qui contrôlent des établissements hors région. On peut citer la présence de centres de décisions de grands groupes comme Auchan, Roquette, 3 Suisses, Décathlon, Bonduelle ou encore Arc International. Ces têtes de groupe présentes dans la région contrôlent ainsi plus de 130000 emplois dans les autres régions françaises et plus de 100000 emplois à l'étranger. Mais, inversement, la région abrite aussi des établissements contrôlés par des groupes dont le siège est hors région. Ainsi, 340000 salariés dépendent d'un centre de décision situé en dehors de la région, soit quand même $44 \%$ des salariés du secteur privé marchand. Parmi eux, $32 \%$ dépendent d'un centre de décision situé dans une autre région française et $12 \%$ d'un centre de décision situé à l'étranger. Le développement de la région n'est donc pas uniquement dans les mains des acteurs présents sur le territoire.

\section{Macroéconomie du Nord-Pas-de-Calais : tirée par l'extérieur ou poussée par l'intérieur?}

La théorie macroéconomique est très souvent mobilisée pour analyser les trajectoires de développement des États ou même d'ensembles plus grands comme l'Union européenne, la catégorie 
des pays émergents, ou même l'économie mondiale dans son ensemble. L'analyse macroéconomique est beaucoup moins souvent convoquée pour discuter des dynamiques territoriales à un échelon infranational. Même si la délimitation d'un espace macroéconomique à un espace administratif a quelque chose d'arbitraire, la construction d'un cadre théorique macroéconomique peut pourtant s'avérer très utile pour éclairer l'histoire et l'avenir d'une région.

La macroéconomie distingue trois étapes du processus économique : 1 / la production de biens et services, 2 / la distribution de revenus, 3/ la dépense des revenus. Le cœur de la macroéconomie pourrait se résumer à la question de savoir si la dépense des revenus ex post est cohérente avec les décisions de production prises ex ante. On va le voir, certaines théories privilégient une approche par la production, d'autres se penchent plutôt sur les revenus.

\subsection{Se représenter l'économie régionale avec la théorie du multiplicateur keynésien}

Une première manière de cerner le développement territorial est de développer à son niveau d'analyse les outils disponibles pour des études macroéconomiques nationales. Il est ainsi possible de produire une représentation de la macroéconomie régionale à l'aide des outils de la comptabilité nationale. Cette approche a été explorée dans le cadre d'une recherche conduite par le Conseil régional Nord-Pas-de-Calais et le laboratoire Clersé de l'Université Lille 1 (Charles et al., 2009).

L'idée directrice était de construire une maquette de la macroéconomie régionale de la même manière qu'il existe une maquette de la macroéconomie nationale. On pourrait ainsi retrouver les grands agrégats traditionnels, et notamment celui du Produit Intérieur Brut. L'ambition peut être poussée plus loin, puisqu'il n'est pas interdit de chercher à mettre en évidence certains concepts macroéconomiques traditionnels, mais au niveau territorial. Le multiplicateur keynésien, qui désigne le supplément de croissance économique généré par un surcroît de dépenses publiques, est de ceux-là. L'intérêt pour ce genre de concept est d'autant plus grand qu'il permet à la puissance publique d'avoir une idée de son impact sur la conjoncture régionale en mesurant par anticipation l'efficacité de son action. 
La difficulté principale que l'on rencontre quand on cherche à établir un multiplicateur keynésien à l'échelle régionale est la disponibilité des données statistiques. La formule réduite pour le calculer nécessite de connaître deux paramètres : d'une part, la propension à épargner, qui désigne la part d'une augmentation de revenu consacrée à l'épargne ; d'autre part, la propension à importer, qui se définit comme le supplément d'importations généré par une augmentation de revenu. La première propension est délicate à estimer, les statistiques de dépenses de consommation n'étant pas toujours cohérentes entre elles. Mais la propension à importer est encore plus difficile à estimer. Au niveau national, est considérée comme importation une marchandise qui provient d'un autre pays. À notre niveau régional, une marchandise en provenance d'Île-deFrance est une importation. L'analyse économique impose de délimiter le périmètre du commerce extérieur aux frontières de la région. En la matière, les statistiques disponibles sont rares. Il a donc été nécessaire de compenser le manque de données par une pluralité de méthodes, de manière à recomposer des estimations des importations en provenance des autres régions françaises. Sans reprendre ici l'ensemble des stratégies employées (voir Charles et al., 2013), l'estimation des échanges interrégionaux repose sur le croisement de plusieurs sources convergentes vers un résultat moyen retenu in fine comme base pour le calcul du multiplicateur.

L'ensemble des grandeurs macroéconomiques sont reprises dans le tableau 2 qui compare les maquettes régionale et française pour l'année 2006, dernière année disponible à l'époque de la rédaction du rapport.

Le premier constat que l'on peut faire concerne les dépenses d'investissement, souvent considérées comme le moteur du développement (faute de données, cet investissement globalise à la fois l'investissement des ménages, des entreprises et des administrations publiques). Elles sont moins intenses en région $(16,5 \% \mathrm{du}$ PIB) qu'à l'échelle nationale (20,6 \% du PIB). La consommation des ménages étant quant à elle plus élevée en région qu'en moyenne nationale, cela laisse à penser que l'investissement des ménages serait moindre dans le Nord-Pas-de-Calais : dépensant beaucoup en consommation, les ménages nordistes n'auraient pas les moyens d'investir. De même, le niveau d'investissement des administrations publiques locales est plus faible qu'en moyenne nationale : 
les collectivités du Nord-Pas-de-Calais consacrent ainsi près de $20 \%$ de leur budget à l'investissement contre $22 \%$ pour l'ensemble des régions. Toutefois, l'absence d'informations sur les dépenses d'investissement de l'État en région et sur l'investissement des entreprises ne permet pas d'appréhender correctement la question de l'investissement dans le Nord-Pas-de-Calais.

Tableau 2. Maquette de la macroéconomie régionale

\begin{tabular}{l|rr|rc}
\hline & \multicolumn{2}{|c}{ France } & \multicolumn{2}{c}{ Nord-Pas-de-Calais } \\
\hline PIB & Mds $€$ & $\%$ du PIB & Mds $€$ & $\%$ du PIB \\
\hline Importations internationales & 1806,4 & 100,0 & 91,0 & 100,0 \\
Importations de France & 506,6 & 28,0 & 37,8 & 41,5 \\
Total des ressources & & & 24,0 & 26,4 \\
Consommation des ménages & 2313,0 & 128,0 & 152,8 & 167,9 \\
Investissement dont APU & 1001,9 & 55,4 & 54,6 & 60,0 \\
Dépenses des APU & 373,2 & 20,6 & 15,0 & 16,5 \\
Dépenses ISBLSM & 422,3 & 23,4 & 21,4 & 23,5 \\
Variation des stocks & 24,2 & 1,4 & nd & nd \\
Exportations internationales & 483,0 & 26,7 & nd & nd \\
Exportations vers la France & & & 37,8 & 41,5 \\
\hline Total des emplois & $\mathbf{2 3 1 3 , 0}$ & $\mathbf{1 2 8 , 0}$ & $\mathbf{1 5 2 , 8}$ & $\mathbf{1 6 7 , 9}$ \\
\hline
\end{tabular}

Légende : APU : Administrations publiques; ISBLM : Institution sans but lucratif au service des ménages; nd : non disponibles.

Source : Insee-Estimation Clersé, en partenariat avec le Conseil régional Nord-Pas-de-Calais.

L'économie régionale se caractérise ensuite par un poids plus important de la consommation des ménages (60\% du PIB contre $55,4 \%$ du PIB à l'échelle nationale). Conformément aux hypothèses keynésiennes traditionnelles, la propension à consommer des habitants du Nord-Pas-de-Calais est ainsi plus élevée dans une région où les revenus sont globalement plus faibles. Cette importance de la consommation régionale renforce la valeur du multiplicateur : une propension à consommer élevée signifie que la propension à épargner est faible, et donc que les revenus distribués sur le territoire vont réalimenter une demande supplémentaire plutôt que de constituer une épargne, sorte de fuite à l'intérieur du circuit macroéconomique régional. De prime abord, cette force consumériste pourrait donc constituer un atout pour la région, la propension à consommer se traduisant par une part importante de la richesse réintroduite dans l'économie locale. Ce serait là une 
lecture trop rapide. La propension à consommer élevée dit simplement qu'une part importante des revenus est consommée, mais pas nécessairement que ces dépenses seront consommées à l'intérieur de l'économie régionale. Le véritable enjeu est donc de distinguer la part de la consommation locale de celle qui s'échappe vers l'extérieur, que ce soit vers d'autres régions ou hors du territoire national.

Troisième constat, encore plus frappant, l'économie du NordPas-de-Calais se caractérise par un degré d'ouverture très important vis-à-vis de l'extérieur, et plus particulièrement des pays étrangers. Les nombreuses importations régionales sont autant de dépenses qui stimulent la demande extérieure au lieu de soutenir la production locale. Cela ne signifie pas que toute politique de distribution de revenus soit inefficace à relancer l'économie, mais qu'elle contribue surtout à dynamiser l'économie des régions et pays voisins. Mais cette ouverture implique également une demande extérieure captée par le territoire, alimentant la production locale. En 2014, les importations ont représenté 40,6 milliards d'euros et les exportations 32,7 milliards: le Nord-Pas-de-Calais se trouve donc certes en situation de déficit commercial, mais il se classe au $4^{\mathrm{e}}$ rang des meilleures régions exportatrices françaises (Bunel, 2015).

Atouts et faiblesses de l'économie régionale se retrouvent finalement dans la formule simplifiée du multiplicateur keynésien. Il est en effet possible d'écrire la valeur ajoutée régionale $(Y)$ comme la somme de la consommation des ménages $(C)$, de l'investissement $(I)$, de la dépense publique $(G)$ et de la demande extérieure $(X-M)$.

$$
Y=C+I+G+(X-M)
$$

De là, on peut simplement écrire que la consommation et la demande d'importations sont des fonctions croissantes du revenu régional, avec $c$ représentant la propension à consommer, $m$ la propension à importer, et $C_{0}$ et $M_{0}$ indiquant des composantes autonomes :

$$
\begin{gathered}
C(Y)=C_{0}+c Y \\
M(Y)=M_{0}+m Y
\end{gathered}
$$

En intégrant ces fonctions de comportement dans la définition du PIB régional, et en supposant dans un premier temps que l'investissement, la dépense publique et les exportations sont autonomes, il vient :

$$
Y=\left(C_{0}+c Y\right)+I_{0}+G_{0}+\left[X_{0}-\left(M_{0}+m Y\right)\right]
$$


Il est alors aisé d'écrire la formule simplifiée du multiplicateur keynésien :

$$
Y=\frac{1}{1-c+m}\left(C_{0}+I_{0}+G_{0}+X_{0}-M_{0}\right)
$$

Si la valeur des deux propensions est importante pour déterminer la taille du multiplicateur, le niveau du PIB dépend surtout de la demande autonome. Toutes choses égales par ailleurs, la production régionale est plus élevée si la propension à consommer $(c)$ est élevée, si la propension à importer $(m)$ est faible, et si les composantes autonomes de la demande (variables indicées 0 ) sont élevées. Du côté de ses faiblesses, la région Nord-Pas-de-Calais souffre d'une propension à importer élevée et d'une faiblesse des dépenses publiques et des investissements. Du côté de ses atouts, la région peut compter sur une forte propension à consommer et des exportations significatives.

Selon cette première approche macroéconomique, le développement de la région passerait par une relance de la dépense publique et des investissements, ainsi que, dans une optique mercantiliste ${ }^{1}$, par une extension des exportations et une diminution des importations. Pour ce tout dernier moyen, la puissance publique dispose d'un levier intéressant en région : la dépense publique affiche un très faible contenu en importations ${ }^{2}$, ce qui permet de faire tourner à plein régime le multiplicateur au premier tour de dépenses. Du point de vue macroéconomique, il est préférable de donner un euro à la puissance publique puisqu'elle le dépensera à $95 \%$ dans l'économie locale, alors que confier un euro à un ménage occasionnera d'une part de l'épargne pour une partie et d'autre part des dépenses à seulement $40 \%$ sur le territoire régional.

L'économie régionale n'est pas totalement soumise à des évolutions des marchés mondiaux qui dépassent le champ d'action des acteurs du territoire. Elle dispose d'une certaine autonomie que les

1. Il convient de noter ici que le développement par l'excédent commercial n'est pas généralisable à l'ensemble des régions simultanément. C'est ce qu'avaient compris les mercantilistes de la Renaissance pour qui les gains d'une nation sont les pertes des autres, le commerce international étant un jeu à somme nulle. La même contrainte s'impose au niveau régional : une région qui réaliserait d'importants excédents commerciaux vers d'autres régions françaises aurait pour contrepartie des régions en déficit commercial.

2. Voir sur le cas français, Heitz et Rini (2006). Plus récemment, des estimations ont été proposées sur les pays de l'OCDE, confirmant le faible contenu en importations de la dépense publique : voir Bussière, Callegari, Ghironi, Sestieri, Yamano (2013). 
décideurs publics peuvent exploiter. La relance des dépenses publiques est plus que jamais nécessaire, notamment en période de ralentissement économique puisque les dernières études sur la valeur du multiplicateur montrent qu'il est plus élevé en période de récession (Creel, Heyer, Plane, 2011).

\subsection{La théorie de la base économique ou l'importance du revenu monétaire capté de l'extérieur}

Quelle que soit l'échelle d'analyse, le PIB peut être appréhendé de trois manières différentes. Sous l'angle de la somme des valeurs ajoutées, il représente le dynamisme productif du territoire. On l'a vu avec le multiplicateur, le PIB peut aussi être interprété comme la somme des dépenses dans l'économie. Mais le PIB peut aussi désigner la somme des revenus: ceux des travailleurs (salaires), ceux des détenteurs du capital (profit), ainsi que certains de ceux de l'État (impôts à la production). C'est cette approche que privilégie la théorie de la base réinterprétée par Laurent Davezies (2009 et 2010).

À l'origine, la théorie de la base économique avait été développée dans les années 1950 par des auteurs anglo-saxons pour traiter de la question du développement territorial par les flux de revenus, avant d'être transformée par un recentrage sur les questions d'emploi et de valeur ajoutée. L'apport de Laurent Davezies consiste à retrouver l'origine de la théorie en reprenant le chemin d'une analyse des revenus. Le principal problème de l'analyse par la valeur ajoutée, c'est qu'elle oublie que si la production se réalise sur un territoire, la distribution de revenus peut se faire sur d'autres territoires. Si les exportations d'un site industriel peuvent être comptabilisées comme une performance du territoire d'implantation, les revenus générés par ce site ne retombent pas tous forcément sur le territoire : les profits générés peuvent être canalisés vers des actionnaires étrangers, les salaires versés peuvent être distribués à des travailleurs "navetteurs ", qui habitent eux aussi sur un autre territoire. En résumé, de bonnes performances à l'exportation ne garantissent pas un bon niveau de développement.

Pour la théorie de la base, le développement local dépend beaucoup de ce qui est appelée "l'économie présentielle », c'est-à-dire des revenus que réussit à capter un territoire, que ces revenus aient été créés sur le territoire ou ailleurs. La théorie de la base de Laurent Davezies est bel et bien d'ordre macroéconomique : elle essaie de 
tenir ensemble les trois dimensions "production de biens et services/distribution des revenus/dépenses des revenus ». Quand on regarde un territoire, certaines personnes peuvent y être présentes à un moment donné, sans y habiter. C'est le cas des touristes de passage qui vont consommer des biens et des services sur le territoire le temps de leur passage. Ces dépenses stimulent la production locale, alors que les revenus de ces consommateurs n'étaient pas issus de ce territoire.

Dans son analyse, Laurent Davezies classe les revenus des territoires en quatre grandes familles, correspondant à quatre types de bases économiques : productif, résidentiel, public, social.

- la base productive est composée des revenus des unités de production tournées vers l'exportation. Par exemple, il s'agit des salaires des travailleurs de l'industrie exportatrice ;

- la base résidentielle provient de l'offre résidentielle. Il s'agit de revenus qui ont été créés hors du territoire, mais dont la résidence est, temporairement ou non, située sur le territoire régional. Les revenus concernés sont ceux des touristes, mais aussi les salaires des travailleurs frontaliers ou les prestations reçues par les retraités résidents ;

- la base publique correspond aux revenus touchés par les fonctionnaires (État, collectivités, hôpitaux). Ces revenus correspondent en partie à une production réalisée sur le territoire, mais il s'agit d'une production de services publics, c'està-dire d'une production non marchande ;

- la base socio-médicale comprend l'ensemble des prestations sociales (allocations diverses: chômage, logement, famille, ...), ainsi que les remboursements des soins de santé.

En moyenne nationale, Davezies (2010) considère que la base productive ne représente que $20 \%$ des revenus d'un territoire, contre $40 \%$ pour la base résidentielle, $20 \%$ pour la base publique, et $20 \%$ encore pour la base socio-médicale (Davezies, 2009). Voilà d'où viennent les revenus.

Mais le réel enjeu macroéconomique est de regarder dans quelle mesure sont dépensés ces revenus. L'économie "présentielle » désigne la capacité d'un territoire à capter au niveau local la dépense de ces différents types de revenus. Pour maximiser le développement local, on en reviendrait finalement à la logique 
keynésienne: d'une part, minimiser les deux fuites du circuit macroéconomique que sont l'épargne et l'importation (ce qui revient à maximiser la taille du multiplicateur); d'autre part, augmenter la demande autonome en comptant sur les apports des exportations et de la demande publique. Si le vocabulaire keynésien fait peur, on peut parfaitement reprendre cela dans les termes de la théorie de la base. D'une part, augmenter le multiplicateur revient à promouvoir les productions locales qui répondent à une demande locale. Ainsi, on évite que les revenus présents sur le territoire ne finissent par être dépensés sur d'autres territoires. D'autre part, la stimulation de la demande autonome peut se traduire par les tentatives pour attirer la localisation des services publics, des touristes, des retraités ou des entreprises exportatrices sur son territoire.

De même qu'une économie peut avoir un niveau de PIB élevé malgré un multiplicateur faible, un territoire peut avoir un niveau de développement élevé malgré une base productive anémiée. Dans le premier cas, il suffirait de compenser par une demande autonome importante. Dans le second cas qui va nous intéresser ici, il convient de compenser par de fortes bases publique, résidentielle et socio-médicale, ainsi que par une forte propension à dépenser localement ces revenus. Telle serait la recette du développement sans l'exportation. Le versant de la pièce, c'est qu'il existe aussi la possibilité de connaître un territoire fortement exportateur, mais faiblement développé. Les raisons tiennent à ce que les autres bases peuvent peiner à fournir les revenus nécessaires à l'économie locale (l'État, les touristes, les retraités, ... ne sont pas présents sur le territoire) et/ou que les revenus de la base productive sont dépensés hors du territoire (les salariés et les actionnaires de l'industrie exportatrice habitent et consomment hors du territoire). L'existence de ces quatre bases distinctes donne naissance à une multitude de situations pour les territoires régionaux.

Selon la classification de Laurent Davezies (2010), les modèles prédominant au sein de la région Nord-Pas-de-Calais sont les modèles «social » et "public et social » traduisant l'importance des difficultés sociales au sein des zones de la région (carte 1$)^{3}$.

3. Région Nord-Pas-de-Calais (2011). 
Carte 1. Typologie des principaux moteurs du développement dans les zones d'emploi

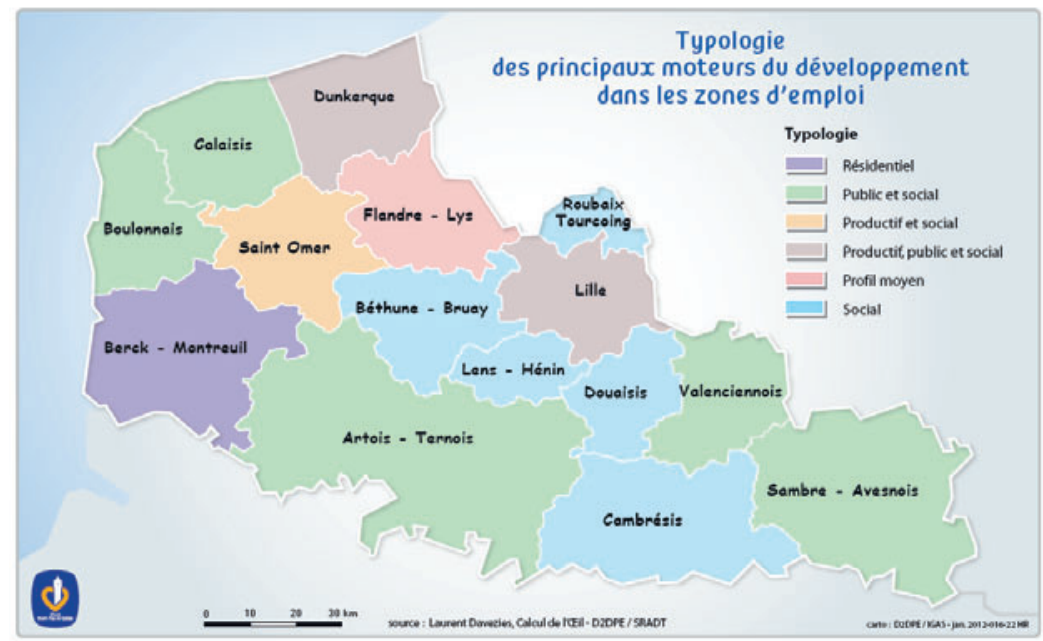

Source : L. Davezies, calculs de l'CFil, D2DPE, IGAS, janvier 2012.

Dans les cinq zones classées dans la catégorie sociale (Roubaix Tourcoing, Béthune-Bruay, Lens-Hénin, le Douaisis et le Cambrésis), le poids de la base sociale dépasse $29 \%$ de la base économique de la zone alors qu'il est de $22,8 \%$ en moyenne nationale. Dans les cinq zones classées dans la catégorie «publique et sociale» (Artois, Boulonnais, Calaisis, Valenciennois, Sambre Avesnois), le poids de la base sociale est également supérieur à $25 \%$. Pour ces zones, la base publique représente également plus de $12 \%$ de la base économique du territoire.

La région Nord-Pas-de-Calais apparaît comme la région dépendant le plus de la base sociale avec en moyenne pondérée $28 \%$ de la base économique des zones d'emploi de la région qui appartiennent à la base sociale. Dans un contexte de crise, cette base sociale peut paradoxalement être vue comme un contexte de stabilité dans le sens où ces revenus ne sont pas impactés à court terme par la récession. Ils constituent toutefois un contexte de fragilité dans la mesure où le niveau des prestations dépend le plus souvent de politiques nationales et où ils traduisent la présence importante de population en difficulté sociale.

En prenant la région dans sa globalité, l'ensemble des autres bases ne sont pas en proportion plus présentes qu'à l'échelle nationale. 
Pour la région Nord-Pas-de-Calais, seules trois zones d'emploi ont un revenu dépendant de façon importante de la base productive qui est a priori la plus exposée dans un contexte de crise économique : la zone de Saint-Omer selon un profil productif et social et les zones d'emploi de Lille et Dunkerque selon un profil productif, public et social traduisant finalement une faible part de la base résidentielle au sein de ces deux territoires.

Concernant la base résidentielle, la région a une proportion plus faible de revenus liés aux dépenses touristiques mais également aux retraites. Sur ce dernier point, quatre zones ont toutefois un poids plus élevé des retraites dans la base économique: la Sambre-Avesnois, le Cambrésis, le Valenciennois et Lens-Hénin.

La zone d'emploi de Berck-Montreuil fait exception grâce au poids important des dépenses touristiques dans les revenus irriguant le territoire.

\subsection{Les activités présentielles comme levier de développement}

La sphère présentielle, en tant qu'ensemble des activités économiques spécifiquement tournées vers la réponse aux besoins des populations résidentes ou touristiques d'une zone, est aujourd'hui un moteur important du développement de l'économie locale. S'appuyant majoritairement sur les services, que ce soit par exemple les services à la personne ou l'économie sociale et solidaire, les activités présentielles s'implantent là où la demande des populations est forte et sont ainsi peu dépendantes des évolutions externes à la zone ou de la conjoncture économique. Leur développement est également moins soumis aux aléas économiques que d'autres types d'activités, qui sont pour la plupart potentiellement délocalisables. Via leur dimension humaine et sociale, les activités présentielles présentent un certain nombre d'avantages pour le développement de territoires au dynamisme modéré sur la scène économique. Ces activités sont toutefois plus souvent génératrices d'emplois moins qualifiés et moins rémunérateurs que la sphère productive.

En termes de niveaux, les emplois présentiels sont le plus développés dans le Montreuillois bénéficiant d'une vocation résidentielle et touristique (Carte 2). Ce type d'emploi est relative- 
ment présent dans les territoires de Lens-Hénin et du Calaisis. À l'inverse, l'économie présentielle est la moins développée dans l'Audomarois qui reste marqué par une forte vocation industrielle et dans la zone de Roubaix-Tourcoing.

Carte 2. L’emploi présentiel dans la région Nord-Pas-de-Calais

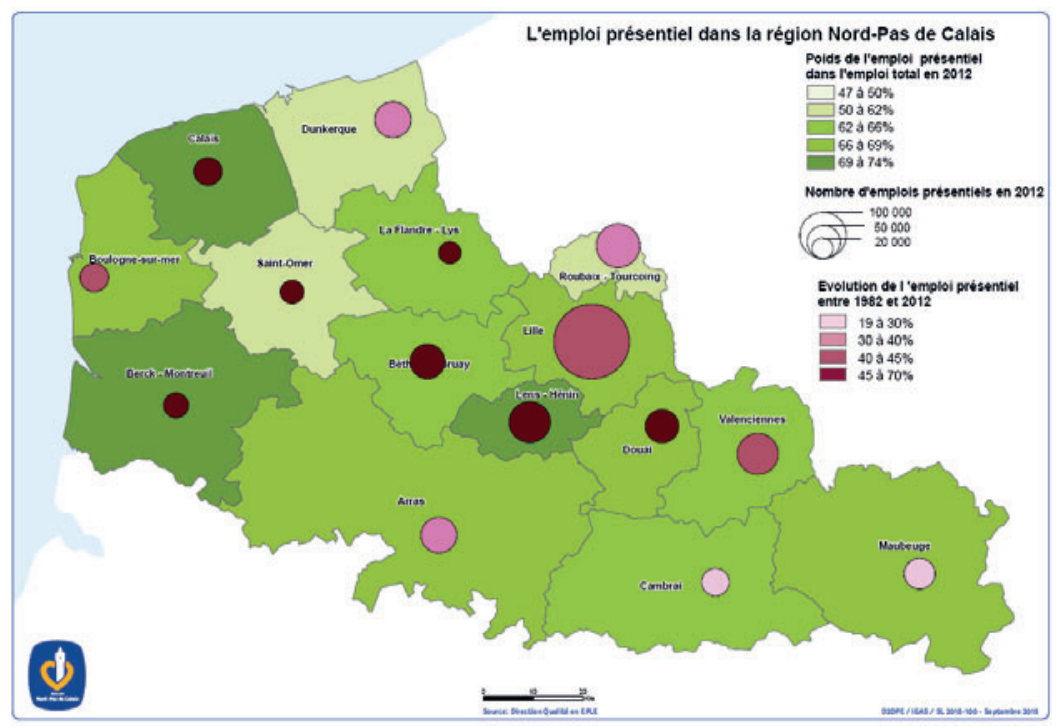

Source : D2DPE, septembre 2015.

Si l'économie présentielle pèse le même poids qu'en moyenne nationale dans l'économie du Nord-Pas-de-Calais, elle est toutefois moins développée que dans la plupart des autres régions en la rapportant au nombre d'habitants. En rapportant le nombre d'emplois présentiels à la population de personnes résidentes selon le recensement de la population, 24 emplois présentiels sont présents pour 100 habitants du Nord-Pas-de-Calais alors que le nombre est de 27 en France métropolitaine. Ce niveau fait du Nord-Pas-de-Calais la deuxième région où cette sphère d'activité est la moins présente devant la Picardie.

Les études menées sur l'impact de cet essor de l'économie présentielle sur le développement des territoires mettent en évidence ces effets positifs pour renforcer les aménités de ces territoires mais pointent également un décalage entre les dynamiques de créations d'emplois et la persistance du chômage dans ces 
mêmes zones d'emploi. Elles mettent également en évidence un manque de qualité des emplois créés dans ces secteurs avec des enjeux de qualification et de structuration de ces nouvelles filières. Favoriser le développement d'une économie présentielle de qualité passe également par une éducation du consommateur. Le consommateur doit ainsi être acteur du développement économique en prenant conscience de l'impact de ses choix individuels sur le modèle de développement.

Encadré 1. L'importance de développer l'économie présentielle face à la vulnérabilité de l'économie productive aux chocs internationaux

Déterminer le degré de vulnérabilité des zones d'emploi de la région Nord-Pas-de-Calais aux aléas économiques repose sur plusieurs facteurs à prendre en considération qui sont divers et parfois non quantifiables (Insee, 2009). La structure du tissu productif peut constituer une source de fragilités face à des événements imprévisibles tels qu'un retournement conjoncturel à court terme ou que l'apparition d'une nouvelle technologie ayant des conséquences à plus long terme. Une zone fortement spécialisée dans un secteur d'activité ou qui concentre la majeure partie de son emploi dans un faible nombre d'établissements est plus exposée lorsqu'un choc économique touche le ou les secteurs concernés.

Carte 3. Typologie sur la capacité d'adaptation des zones d'emploi aux chocs économiques

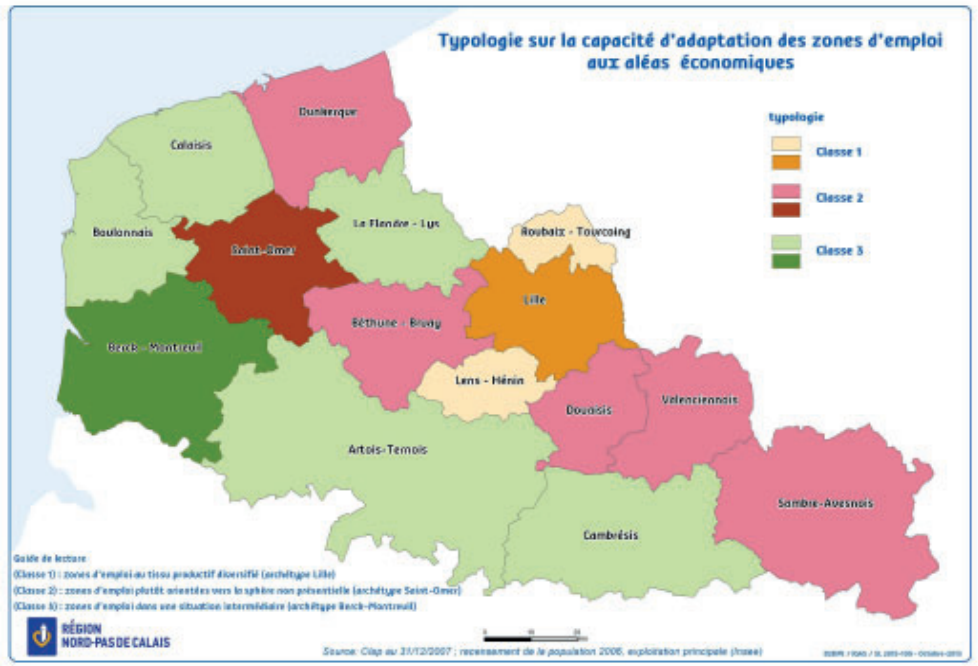

Source: 
La surreprésentation d'un secteur d'activité et la concentration spatiale d'une activité dans l'emploi local, sources de performance lorsque la conjoncture est favorable, peuvent se transformer en faiblesse en cas de retournement conjoncturel. Face à ces vulnérabilités, la capacité d'adaptation d'un territoire peut dépendre fortement du niveau de formation des actifs et des caractéristiques de la main-d'œuvre ainsi que de l'état du marché du travail.

À l'échelle infrarégionale, les territoires ne présentent pas tous les mêmes caractéristiques. Ainsi, des zones d'emploi de Lens-Hénin, Lille ou Roubaix-Tourcoing ont des indices de spécialisation et de concentration faibles. À l'inverse, des zones comme Berck-Montreuil ou SaintOmer ont des indices beaucoup plus élevés, ce qui est susceptible de se traduire par une plus grande fragilité face aux aléas économiques.

\subsection{Une lecture transversale des profils économiques régionaux}

Une étude réalisée par l'Insee, l'État et la Région Nord-Pas-deCalais (2011 et 2012) a permis d'élaborer, au-delà des délimitations administratives traditionnelles, une carte de synthèse des profils économiques visant à dessiner une nouvelle géographie régionale à partir de systèmes économiques cohérents assurant des fonctions économiques (carte 4).

Autrement dit, de grands types d'espaces en Nord-Pas-de-Calais sont identifiés au regard des problématiques économiques locales. L'objectif de cette étude était d'expliquer la constitution actuelle du tissu productif par la rétrospective des mutations subies par les territoires. Le potentiel de développement des espaces peut également se trouver dans les fonctions occupées par les emplois ou l'importance des activités présentielles. Le dynamisme économique y est également abordé à travers l'attractivité des zones et leur capacité à faire face aux chocs conjoncturels.

Cette carte de synthèse s'appuie sur l'évolution de la structure productive, la fonction des emplois, la trajectoire de la sphère présentielle, l'attractivité économique et enfin l'exposition aux aléas économiques. Le croisement de ces indicateurs fait émerger huit espaces régionaux au regard de la structure et des dynamiques économiques des territoires.

Le premier espace qui émerge de l'analyse des polarités et des mutations économiques regroupe l'ensemble de la métropole lilloise au Lensois et à l'Arrageois. Cet ensemble se caractérise par 
une grande attractivité économique, une sphère présentielle plus développée, un tissu productif diversifié et une présence des fonctions métropolitaines supérieures et des fonctions de distribution ou de transport-logistique.

Carte 4. Les espaces régionaux au regard des mutations et polarités économiques

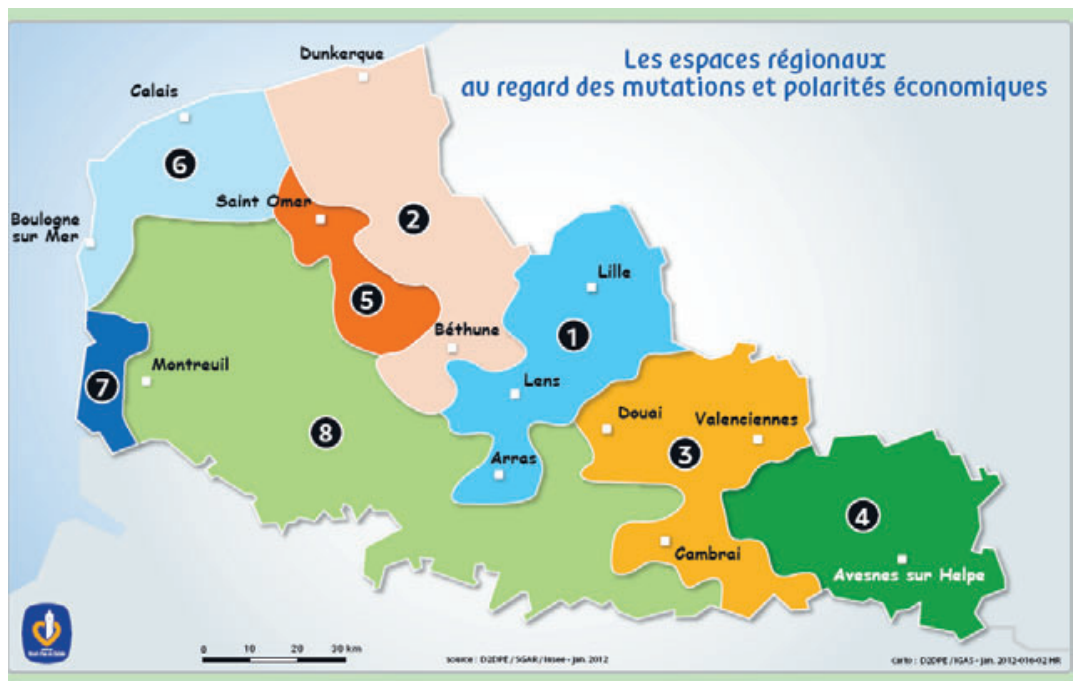

Source : D2DPE, janvier 2012.

De part et d'autre de ce territoire s'étendent deux espaces marqués par l'importance de l'industrie, par une plus grande sensibilité aux aléas économiques et par un développement récent du secteur tertiaire. L'espace situé au Nord qui s'étend de Dunkerque à la frontière lilloise en englobant le Béthunois est de plus caractérisé par une moindre part de l'emploi présentiel. L'espace situé au Sud qui englobe le Douaisis, le Valenciennois et le Cambrésis a une sphère d'emplois présentiels un peu plus développée et apparaît plus attractif économiquement dans sa partie centrale. Au Sud, la Sambre-Avesnois se distingue par un tissu économique plus diversifié malgré la présence encore importante d'industries lourdes. Ce territoire est toutefois moyennement attractif économiquement et sensible aux aléas économiques. Un territoire marqué par l'importance de l'industrie se dessine autour de Saint-Omer en s'étendant au Sud-Est. La sphère présentielle est peu présente au sein de ce territoire qui apparaît parmi les plus exposés aux aléas économiques. 
Le long du littoral, un espace regroupe les zones du Boulonnais et du Calaisis caractérisées par une tertiarisation plus précoce dans les villes-centres qui s'étend progressivement dans le reste du territoire. Cet espace apparaît économiquement peu attractif sur la période récente mais bénéficie d'une proportion d'emplois dans la sphère présentielle proche de la moyenne régionale. Plus au Sud, la zone littorale allant d'Étaples à Berck se distingue par l'importance de l'économie présentielle liée en particulier à l'attractivité touristique de la zone. Ce territoire apparaît peu attractif pour l'économie productive et semble peu exposé aux aléas conjoncturels. Enfin, le dernier espace regroupe l'ensemble des espaces ruraux du Pas-de-Calais en s'étendant jusqu'au sud-ouest du Cambrésis. Ce territoire principalement rural n'apparaît pas attractif d'un point de vue économique et possède encore une part non négligeable d'emplois orientés vers l'agriculture. Les métiers du BTP et les services de proximité y sont également plus présents qu'en moyenne régionale.

Les espaces sont donc aujourd'hui non seulement polarisés par leur structure sectorielle mais aussi par les fonctions occupées par les emplois. Cette organisation spatiale induit des écarts territoriaux importants, que ce soit en termes de dynamisme ou de vulnérabilité, tout en connaissant des rythmes différents de développement humain.

\section{Développement économique et inégalités territoriales : la nécessité de nouveaux indicateurs}

\subsection{Compléter le PIB pour rendre compte des inégalités au sein des territoires}

La richesse produite sur un territoire prend à la fois en compte la rémunération du capital et la rémunération du travail. De fait, elle ne peut donc être assimilée au niveau des revenus dont disposent les habitants de ce territoire. Il y a ainsi des limites à calculer le ratio entre la valeur ajoutée circulant sur un territoire et le nombre de personnes qui y résident. Rien ne dit que toute la population d'un territoire participe de la même manière à la circulation de la richesse. Des régions homogènes peuvent présenter des PIB proches de régions où une population très pauvre côtoie une 
population très riche, présenter des situations très distinctes. Certaines régions comme le Languedoc-Roussillon ou la province de Liège vont, comme le Nord-Pas-de-Calais, connaître une situation peu favorable: taux d'emploi faible, taux de chômage de longue durée élevé et un taux de pauvreté relativement important. À l'opposé, la région allemande de Trier ou la région britannique de Leicestershire sont mieux loties : taux d'emploi élevé, faible taux de chômage de longue durée et taux de pauvreté peu élevé. Enfin, la région espagnole de La Rioja ou la région irlandaise de Border Midland and Western vont afficher une situation très défavorable : taux de pauvreté élevé malgré un taux d'emploi et un chômage de longue durée moyen.

Graphique 3. Un même PIB par habitant mais des profils économiques et sociaux très différents
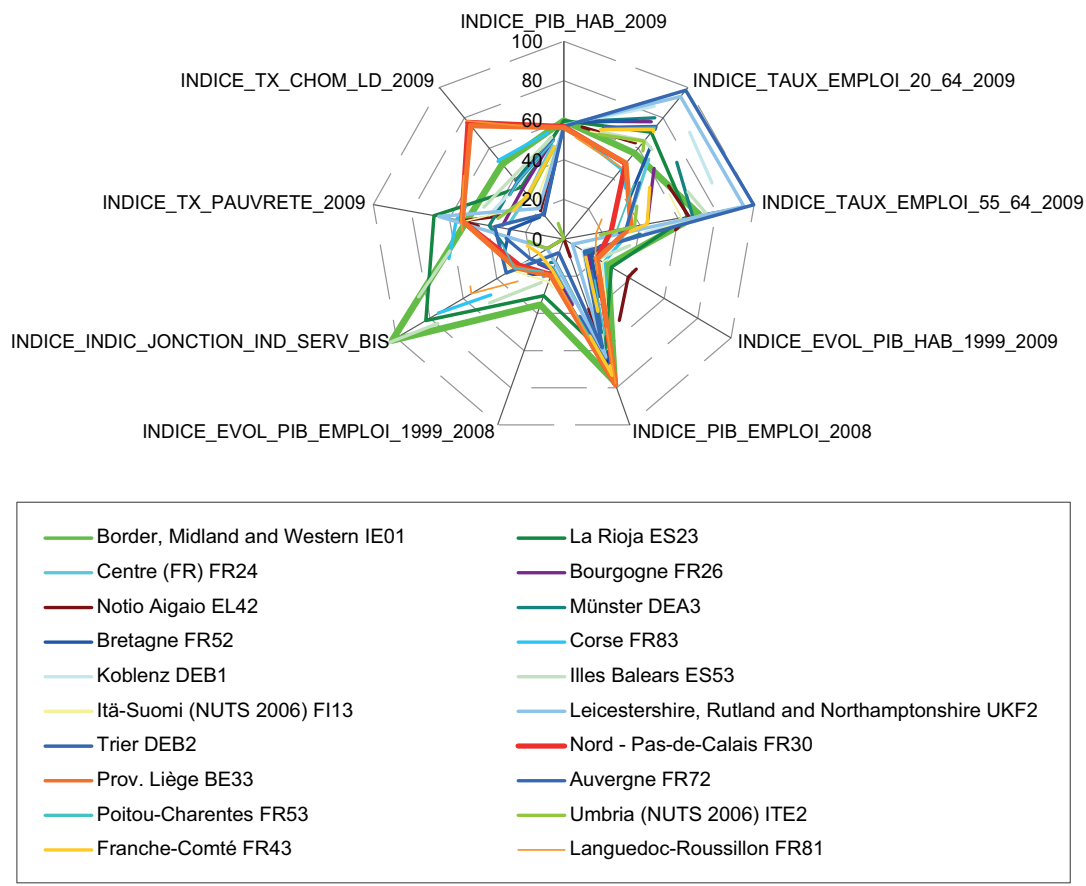

Source: Insee et Conseil Régional Nord-Pas-de-Calais, 2012, « D'autres critères que le PIB pour comprendre les régions françaises », Pages de Profils, $n^{\circ} 121$. 


\subsection{Développement humain et santé sociale}

Le calcul de l'IDH à l'échelle régionale est une première approche pour mesurer l'aspect multidimensionnel du développement (IDH-2) ${ }^{4}$.

L'IDH-2 constitue une déclinaison régionale de l'Indicateur de Développement Humain du PNUD. Il reprend les trois grandes dimensions de l'indicateur du PNUD : la santé, l'éducation et le niveau de vie, à savoir la capacité à bénéficier d'une vie longue et saine, la capacité d'accès à l'éducation et aux connaissances et enfin la possibilité d'accéder aux ressources matérielles indispensables pour atteindre un niveau de vie décent. Les variables retenues sont toutefois différentes à la fois en raison de la disponibilité des données à une échelle infranationale et d'une traduction différente de la dimension à une échelle territoriale. L'IDH2, tel que défini par Aurélien Boutaud, se compose ainsi de l'espérance de vie, de la part de la population diplômée et du revenu fiscal médian par unité de consommation (Annexe 1).

L'IDH-2 reprend ainsi les paramètres de l'indice du PNUD, mais avec des indicateurs plus adaptés à la situation des régions françaises (tableau 3).

Le Nord-Pas-de-Calais est la région française ayant l'IDH-2 le plus faible $(0,695)$. Elle se situe derrière la Picardie $(0,709)$, la Corse $(0,727)$, la Champagne-Ardenne $(0,731)$, et loin derrière RhôneAlpes $(0,807)$ Midi-Pyrénées $(0,807)$, et l'Île-de-France $(0,823)$. Ce résultat s'explique en partie par un gros retard en termes d'espérance de vie : l'espérance de vie à la naissance en Nord-Pas-deCalais est de 75,3 années pour les hommes contre 78,5 années en moyenne pour la France métropolitaine, 82,8 années pour les femmes contre 84,9 en moyenne métropolitaine. La région NordPas-de-Calais a également l'indice niveau de vie le plus faible de toutes les régions françaises $(0,770$ contre 0,845 en moyenne nationale) : le revenu fiscal médian est de 16797 euros pour la région contre 19218 euros pour la France métropolitaine.

La décomposition de l'IDH-2 permet d'illustrer le positionnement différent des régions selon les dimensions. Si la région NordPas-de-Calais est $22^{\mathrm{e}}$ en termes de niveau de vie et de santé, elle est

4. Cette déclinaison a été proposée en 2009 par Aurélien Boutaud. 
un peu moins mal positionnée en termes d'éducation, étant au $18^{\mathrm{e}}$ rang des régions françaises. Le Nord-Pas-de-Calais fait figure d'exception car peu de régions ont des profils similaires sur chaque dimension (tableau 3).

Tableau 3. Disparités régionales selon I'Indice de Développement Humain (IDH-2) en 2011

\begin{tabular}{lcccc}
\hline & \multicolumn{3}{c}{$\begin{array}{c}\text { Dimension } \\
\text { Niveau de vie }\end{array}$} & $\begin{array}{c}\text { Éducation } \\
\text { IDH-2 des } \\
\text { régions }\end{array}$ \\
\hline île-de-France & 0,927 & 0,655 & 0,887 & 0,823 \\
Rhône-Alpes & 0,869 & 0,664 & 0,889 & 0,807 \\
Midi-Pyrénées & 0,836 & 0,700 & 0,884 & 0,807 \\
Aquitaine & 0,841 & 0,688 & 0,871 & 0,800 \\
Bretagne & 0,838 & 0,744 & 0,803 & 0,795 \\
Alsace & 0,832 & 0,674 & 0,862 & 0,789 \\
Provence-Alpes-Côte d'Azur & 0,884 & 0,621 & 0,836 & 0,780 \\
Limousin & 0,841 & 0,632 & 0,864 & 0,779 \\
Centre & 0,814 & 0,699 & 0,824 & 0,779 \\
Auvergne & 0,845 & 0,648 & 0,842 & 0,779 \\
Poitou-Charentes & 0,816 & 0,694 & 0,808 & 0,773 \\
Franche-Comté & 0,815 & 0,653 & 0,840 & 0,769 \\
Bourgogne & 0,839 & 0,646 & 0,820 & 0,769 \\
Languedoc-Roussillon & 0,830 & 0,643 & 0,801 & 0,758 \\
Basse-Normandie & 0,786 & 0,629 & 0,834 & 0,750 \\
Lorraine & 0,813 & 0,606 & 0,818 & 0,746 \\
Haute-Normandie & 0,824 & 0,605 & 0,784 & 0,738 \\
Champagne-Ardenne & 0,836 & 0,581 & 0,783 & 0,734 \\
Corse & 0,816 & 0,592 & 0,784 & 0,731 \\
Picardie & 0,798 & 0,544 & 0,838 & 0,727 \\
Nord-Pas-de-Calais & 0,819 & 0,558 & 0,749 & 0,709 \\
\hline France métropolitaine & 0,770 & 0,605 & 0,709 & 0,695 \\
\hline Envet & 0,845 & 0,649 & 0,840 & $\mathbf{0 , 7 7 8}$ \\
\hline
\end{tabular}

En vert, les valeurs au-dessus de la moyenne métropolitaine; en rouge les valeurs en dessous de la moyenne métropolitaine Sources: Calculs D2DPE, Conseil régional Nord-Pas-de-Calais.

Si l'île-de-France est la première région française en termes de revenus et d'espérance de vie, c'est la Bretagne qui compte la part relative la plus élevée de diplômés dans sa population. Des régions comme Midi-Pyrénées ont même des profils atypiques : $9^{\mathrm{e}}$ région française à la lecture de l'indice du niveau de vie, elle est la $2^{\mathrm{e}}$ région en part de diplômés dans la population non scolarisée et 
la $3^{\mathrm{e}}$ région en termes de santé à la lecture de l'espérance de vie. La situation est inverse pour l'Alsace $: 2^{\mathrm{e}}$ région française en termes de revenus, elle se positionne beaucoup moins bien sur les deux autres dimensions du développement humain : $10^{\mathrm{e}}$ en matière de santé, $15^{\mathrm{e}}$ en matière d'éducation.

Le croisement de l'IDH-2 avec le taux de pauvreté monétaire et le rapport inter-décile des revenus démontre que la région Nord-Pasde-Calais est avec la Corse et le Languedoc-Roussillon l'une des trois régions particulièrement en difficulté sur le plan social : elles sont les trois dernières régions en termes de développement humain et connaissent également des inégalités sociales et une pauvreté particulièrement élevées : leur taux de pauvreté monétaire est supérieur à $18,5 \%$ et elles font partie des cinq régions pour lesquelles le rapport inter-décile est supérieur à la moyenne métropolitaine.

La comparaison avec les autres régions françaises montre que performance économique n'implique pas nécessairement un développement humain élevé (tableau 4). Si l'île-de-France et RhôneAlpes combinent un fort PIB par habitant et un IDH élevé, il n'en est pas de même pour la Provence-Alpes-Côte-d'Azur, la HauteNormandie et la Champagne-Ardenne, respectivement $8^{\mathrm{e}}, 18^{\mathrm{e}}$ et $19^{\text {e }}$ à la lecture de l'IDH régionalisé, alors qu'elles sont parmi les 9 premières régions françaises en termes de richesse économique. L'Île-de-France est néanmoins caractérisée par des inégalités de revenus très fortes : le rapport $\mathrm{D} 9 / \mathrm{D} 1^{5}$ est égal à 7,5 , ce qui signifie que le revenu minimum des $10 \%$ les plus riches est plus de 7 fois supérieur au revenu maximum des $10 \%$ les plus pauvres. A contrario, les régions Midi-Pyrénées et Limousin sont relativement mieux positionnées en termes de développement humain : MidiPyrénées est la $3^{\mathrm{e}}$ région française en termes de développement humain alors qu'elle a la $8^{\mathrm{e}}$ en termes de PIB/habitant. Le contraste pour le Limousin est encore plus fort : $22^{\mathrm{e}}$ région en termes de richesse économique, le Limousin a un IDH régional supérieur à la moyenne nationale. Les bonnes performances de ces deux régions en matière d'éducation expliquent en partie cette différence.

5. Pour mesurer la dispersion des revenus, est utilisé le rapport inter-décile (D9/D1) du revenu fiscal par unité de consommation, qui établit le rapport entre les hauts revenus et les bas revenus. Le seuil des bas revenus correspond au premier décile (D1) : seules $10 \%$ des personnes appartiennent à un ménage qui déclare un revenu par unité de consommation inférieur à cette valeur. Le seuil des hauts revenus correspond au neuvième décile (D9): seules $10 \%$ des personnes appartiennent à un ménage qui déclare un revenu par unité de consommation supérieur à cette valeur. 
Tableau 4. Comparaison des régions françaises en 2011

\begin{tabular}{|c|c|c|c|c|c|c|c|c|}
\hline Région & $\begin{array}{c}\text { PIB/hab } \\
2011 \\
\text { en } €\end{array}$ & $\begin{array}{c}\text { PIB/hab } \\
2011 \\
\text { rang }\end{array}$ & $\begin{array}{c}\text { IDH-2 } \\
2011\end{array}$ & $\begin{array}{l}\text { IDH-2 } \\
\text { rang } \\
2011\end{array}$ & $\begin{array}{c}\text { Taux de } \\
\text { pauvreté } \\
\text { monétaire } \\
2011(\text { en \%) }\end{array}$ & $\begin{array}{c}\text { Taux de } \\
\text { pauvreté } \\
\text { monétaire } \\
\text { rang } 2011\end{array}$ & $\begin{array}{c}\text { D9/D1 } \\
2011\end{array}$ & $\begin{array}{c}\text { D9/D1 } \\
\text { rang } \\
2011\end{array}$ \\
\hline île-de-France & 51850 & 1 & 0,823 & 1 & 13,5 & 9 & 7,5 & 22 \\
\hline Rhône-Alpes & 31784 & 2 & 0,807 & 2 & 12,4 & 4 & 5,2 & 14 \\
\hline Provence-Alpes-Côte d'Azur & 29687 & 3 & 0,779 & 8 & 16,4 & 19 & 6,8 & 20 \\
\hline Alsace & 29318 & 4 & 0,780 & 7 & 12,2 & 3 & 5,2 & 15 \\
\hline Champagne-Ardenne & 28239 & 5 & 0,731 & 19 & 15,5 & 17 & 5,4 & 16 \\
\hline Pays de la Loire & 28195 & 6 & 0,789 & 6 & 11,6 & 2 & 4,0 & 1 \\
\hline Aquitaine & 28085 & 7 & 0,800 & 4 & 13,5 & 8 & 4,8 & 9 \\
\hline Midi-Pyrénées & 27833 & 8 & 0,807 & 3 & 14,6 & 14 & 5,2 & 13 \\
\hline Haute-Normandie & 27511 & 9 & 0,734 & 18 & 13,8 & 11 & 5,0 & 11 \\
\hline Centre-Val de Loire & 26494 & 2 & 0,807 & 2 & 12,4 & 4 & 5,2 & 14 \\
\hline Bourgogne & 26376 & 11 & 0,758 & 14 & 13,2 & 7 & 4,5 & 4 \\
\hline Bretagne & 26340 & 12 & 0,795 & 5 & 11,5 & 1 & 4,1 & 2 \\
\hline Corse & 26162 & 13 & 0,727 & 20 & 19,2 & 20 & 6,6 & 18 \\
\hline Basse-Normandie & 25679 & 14 & 0,746 & 16 & 13,7 & 10 & 4,5 & 3 \\
\hline Auvergne & 25679 & 15 & 0,773 & 11 & 14,3 & 12 & 4,6 & 5 \\
\hline Nord-Pas-de-Calais & 25655 & 16 & 0,695 & 22 & 19,7 & 22 & 6,7 & 19 \\
\hline Poitou-Charentes & 25413 & 17 & 0,769 & 12 & 14,3 & 13 & 4,6 & 6 \\
\hline Franche-Comté & 24929 & 18 & 0,769 & 13 & 13,1 & 6 & 4,7 & 8 \\
\hline Languedoc-Roussillon & 24365 & 19 & 0,750 & 15 & 19,6 & 21 & 7,2 & 21 \\
\hline Picardie & 24170 & 20 & 0,709 & 21 & 15,5 & 18 & 5,4 & 17 \\
\hline Lorraine & 24060 & 21 & 0,738 & 17 & 14,7 & 15 & 5,1 & 12 \\
\hline Limousin & 23528 & 22 & 0,779 & 9 & 15,4 & 16 & 4,8 & 10 \\
\hline France métropolitaine & 31976 & & 0,778 & & 14,3 & & 5,6 & \\
\hline
\end{tabular}

En vert, les valeurs plus favorables qu'en moyenne métropolitaine; en rouge les valeurs moins favorables qu'en moyenne métropolitaine.

Note: Taux de pauvreté monétaire : un individu est considéré comme pauvre lorsqu'il vit dans un ménage dont le niveau de vie est inférieur à $60 \%$ de la médiane des niveaux de vie. Le ratio D9/D1 rapporte le niveau de revenu minimum des $10 \%$ les plus riches au revenu maximum des $10 \%$ les plus pauvres.

Sources : Calculs D2DPE, Conseil régional Nord-Pas-de-Calais. 
La comparaison des résultats entre 1999 et 2011 montre une amélioration générale de la situation en 10 ans (carte 5) : toutes les régions ont vu leur IDH-2 augmenter sur cette période. Avec les définitions et les modes de calculs retenus, l'IDH-2 de la France métropolitaine est passé de 0,667 en 1999 à 0,778 en 2011. Si la région Nord-Pas-de-Calais reste la $22^{\mathrm{e}}$ région française en termes de développement humain, elle semble connaître une dynamique de rattrapage. L'IDH-2 du Nord-Pas-de-Calais est passé de 0,574 à 0,695 entre 1999 et 2011, soit un différentiel supérieur à la moyenne métropolitaine. Cette progression est néanmoins bien inférieure à celles de la Corse, de la Basse-Normandie, d'Aquitaine, de Bretagne et des Pays-de-la-Loire, régions ayant connu les plus fortes augmentations relatives de leur IDH-2 entre 1999 et 2011. Les dynamiques régionales sont sensiblement différentes selon les dimensions du développement humain.

\section{Carte 5. Profil des régions selon leur trajectoire en matière de développement humain}

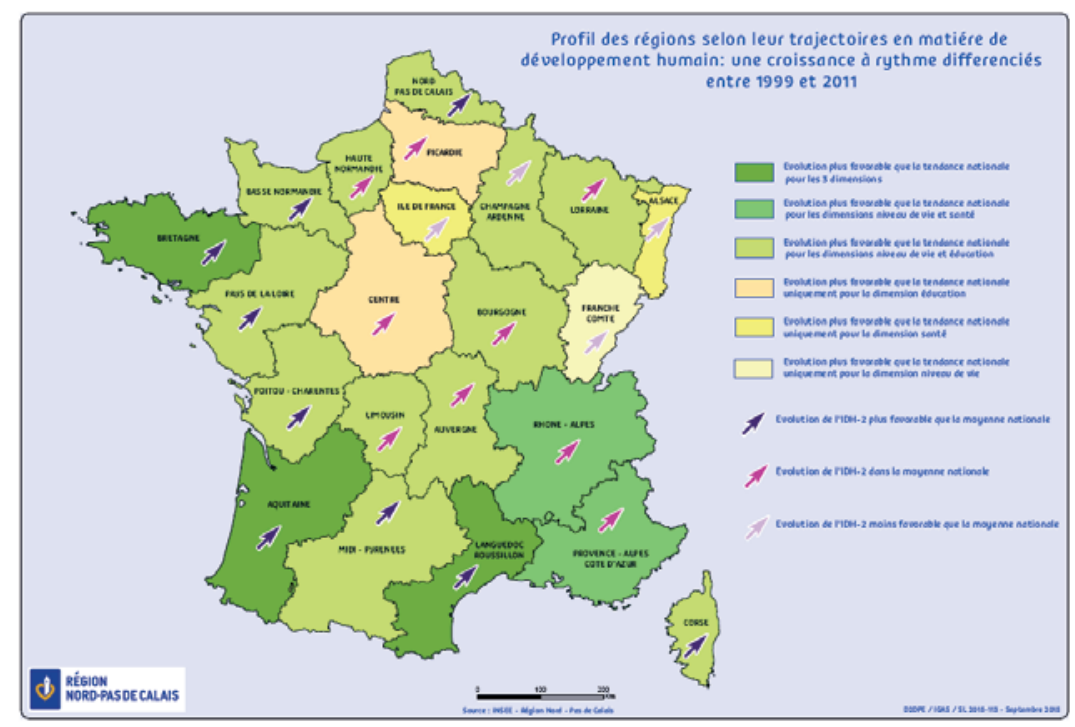

Source : D2DPE, septembre 2015.

La région Nord-Pas-de-Calais connaît l'une des dynamiques les plus importantes en termes d'éducation, derrière notamment les régions Poitou-Charentes, Basse-Normandie et Pays-de-la-Loire qui sont les régions connaissant les hausses relatives du taux de 
diplômés sur leur territoire les plus importantes. Le Nord-Pas-deCalais connaît également une dynamique favorable en termes de niveau de vie, mais beaucoup moins importante que la Corse qui est la région où l'augmentation relative du revenu fiscal médian est la plus forte. L'Île-de-France et l'Alsace ont, à contrario, connu l'évolution la plus faible entre 1999 et 2011 mais ont toujours les revenus fiscaux médians les plus élevés des régions métropolitaines. En revanche, concernant la dimension santé, l'espérance de vie des habitants du Nord-Pas-de-Calais augmente moins rapidement qu'en moyenne nationale. Autrement dit, le retard du NordPas-de-Calais en termes d'espérance de vie s'accentue, alors que la région est déjà celle où l'espérance de vie de ses habitants est la plus faible. L'Alsace, l'Aquitaine et la Bretagne sont les régions connaissant les évolutions les plus favorables en termes d'espérance de vie.

En plus d'un développement humain en retrait, le Nord-Pas-deCalais est marqué par des inégalités importantes. L'indicateur de Santé sociale construit par Florence Jany-Catrice (2009 et 2013) permet de mettre en évidence le caractère multidimensionnel de ces inégalités en reprenant les six dimensions du baromètre des inégalités et de la pauvreté également calculé par Florence JanyCatrice pour la région: éducation, justice, logement, santé, revenus et travail-emploi. À ces dimensions ont été rajoutées deux dimensions permettant de prendre en compte le capital social des régions : le lien social et lien interindividuel (Annexe 2).

Selon cet indicateur, le Nord-Pas-de-Calais est la $22^{\mathrm{e}}$ région de France métropolitaine en matière de santé sociale derrière la Picardie et les deux régions du pourtour méditerranéen : Languedoc-Roussillon et Provence-Alpes-Côte-d'Azur alors que cette dernière région est la quatrième région française en matière de richesse économique (tableau 5). L'IDH-2 et l'ISS mettent ainsi en évidence la non-corrélation entre richesse économique et santé sociale. Cette divergence est confirmée par le cas de l'Île-de-France dont la richesse économique par habitant est nettement supérieure aux autres et qui se classe $17^{\mathrm{e}}$ région au regard de sa santé sociale. À l'inverse, le Limousin est la région ayant la meilleure santé sociale avec un PIB/habitant parmi les moins élevés. Plusieurs variables renvoyant à plusieurs dimensions expliquent le mauvais positionnement de la région Nord-Pas-de-Calais au regard de cet indicateur. 
En premier lieu, la région est pénalisée par des difficultés importantes en matière de santé avec l'espérance de vie la plus faible pour les hommes et parmi les deux plus faibles pour les femmes. En matière de revenus, la région est pénalisée par la part la plus élevée de jeunes de moins de 17 ans vivant dans un ménage sous le seuil de pauvreté et par le plus grand nombre de dossiers de surendettement déposés pour 100000 habitants.

Tableau 5. Positionnement des régions françaises au regard du PIB/habitant, de I'Indicateur de Santé Sociale et de I'IDH-2 en 2008

\begin{tabular}{|c|c|c|c|c|c|c|}
\hline Région & $\begin{array}{c}\text { PIB/hab. } \\
\text { en } €\end{array}$ & $\begin{array}{c}\text { Rang } \\
\text { Pib/hab. }\end{array}$ & ISS & $\begin{array}{c}\text { Rang } \\
\text { ISS }\end{array}$ & IDH-2 & $\begin{array}{l}\text { Rang } \\
\text { IDH-2 }\end{array}$ \\
\hline île-de-France & 47696 & 1 & 48,2 & 17 & 0,788 & 1 \\
\hline Rhône-Alpes & 30513 & 2 & 61,8 & 7 & 0,763 & 3 \\
\hline Provence-Alpes-Côte d'Azur & 28500 & 3 & 43,9 & 19 & 0,735 & 10 \\
\hline Alsace & 28285 & 4 & 65,6 & 5 & 0,742 & 7 \\
\hline Champagne-Ardenne & 27917 & 5 & 51,1 & 16 & 0,687 & 20 \\
\hline Haute-Normandie & 27584 & 6 & 46,6 & 18 & 0,693 & 18 \\
\hline Pays de la Loire & 27357 & 7 & 66,3 & 3 & 0,746 & 6 \\
\hline Aquitaine & 27322 & 8 & 60,9 & 8 & 0,755 & 4 \\
\hline Midi-Pyrénées & 27254 & 9 & 62,1 & 6 & 0,765 & 2 \\
\hline Bretagne & 26530 & 10 & 67,6 & 2 & 0,755 & 5 \\
\hline Bourgogne & 26459 & 11 & 57,7 & 13 & 0,721 & 14 \\
\hline Centre & 26449 & 12 & 59,1 & 11 & 0,736 & 9 \\
\hline Auvergne & 25260 & 13 & 65,9 & 4 & 0,735 & 11 \\
\hline Poitou-Charentes & 25010 & 14 & 59,5 & 10 & 0,733 & 13 \\
\hline Franche-Comté & 24908 & 15 & 60,5 & 9 & 0,733 & 12 \\
\hline Nord - Pas-de-Calais & 24683 & 16 & 33,3 & 22 & 0,651 & 22 \\
\hline Basse-Normandie & 24536 & 17 & 58 & 12 & 0,703 & 16 \\
\hline Lorraine & 24497 & 18 & 53,7 & 15 & 0,696 & 17 \\
\hline Limousin & 24296 & 19 & 71,3 & 1 & 0,742 & 8 \\
\hline Picardie & 23872 & 20 & 38,4 & 21 & 0,664 & 21 \\
\hline Corse & 23803 & 21 & 54,8 & 14 & 0,689 & 19 \\
\hline Languedoc-Roussillon & 23741 & 22 & 42,5 & 20 & 0,711 & 15 \\
\hline France & 30700 & & 53,8 & & 0,737 & \\
\hline
\end{tabular}

Sources : Calculs D2DPE, Conseil régional Nord-Pas-de-Calais. 


\subsection{Des disparités territoriales persistantes}

Si la région Nord-Pas-de-Calais affiche globalement le niveau de développement humain le plus faible des régions de France métropolitaine, les contrastes sont importants à l'échelle infrarégionale. La déclinaison communale de l'indicateur de développement humain (IDH-4) permet de mesurer ces contrastes à l'échelle des territoires (Région Nord-Pas-de-Calais, 2015).

À une échelle territoriale fine, l'IDH4 synthétise un état de développement humain relatif à des populations qui, à un moment donné, y résident, sans nécessairement y inscrire l'ensemble de leur cycle de vie. En effet, les mobilités résidentielles, notamment entre l'enfance et la vie adulte, puis au cours de la vie active, induisent des mécaniques de transformations sociales d'autant plus importantes que l'échelon géographique considéré est fin. De même, les migrations alternantes, entre lieu de résidence et lieu de travail ou d'étude, traduisent une mise en relation croissante des territoires, et une interdépendance dans l'état du développement humain. Parfois, des mécaniques de concentration sociale peuvent majorer l'état dégradé du développement tel que retracé par l'IDH4, en polarisant sur un territoire les populations les plus précaires sur les trois dimensions prises en compte : tout en signalant une problématique pour cet espace, cela ne signifie pas pour autant que d'autres populations venues s'installer dans un tel espace verront leurs opportunités de développement humain nécessairement contraintes.

L'IDH4 à l'échelle des communes retrace plus spécifiquement une concentration, en un espace et à une période donnée, de populations avec un certain degré de ressources monétaires, éducatives et sanitaires.

Le calcul de cet indicateur met en évidence de fortes disparités en matière de développement humain au sein de la région (carte 6).

Ainsi, l'ancien bassin minier forme un espace homogène où l'IDH est très inférieur à la moyenne régionale s'opposant aux communes qui entourent Lille et Arras caractérisées par un niveau de développement nettement supérieur à la moyenne régionale grâce à un niveau de revenu plus élevé, une moindre proportion de non-diplômés et un indice comparatif de mortalité plus faible que la moyenne régionale. 
Carte 6. Déclinaison communale de I'Indice de Développement Humain (IDH-4)

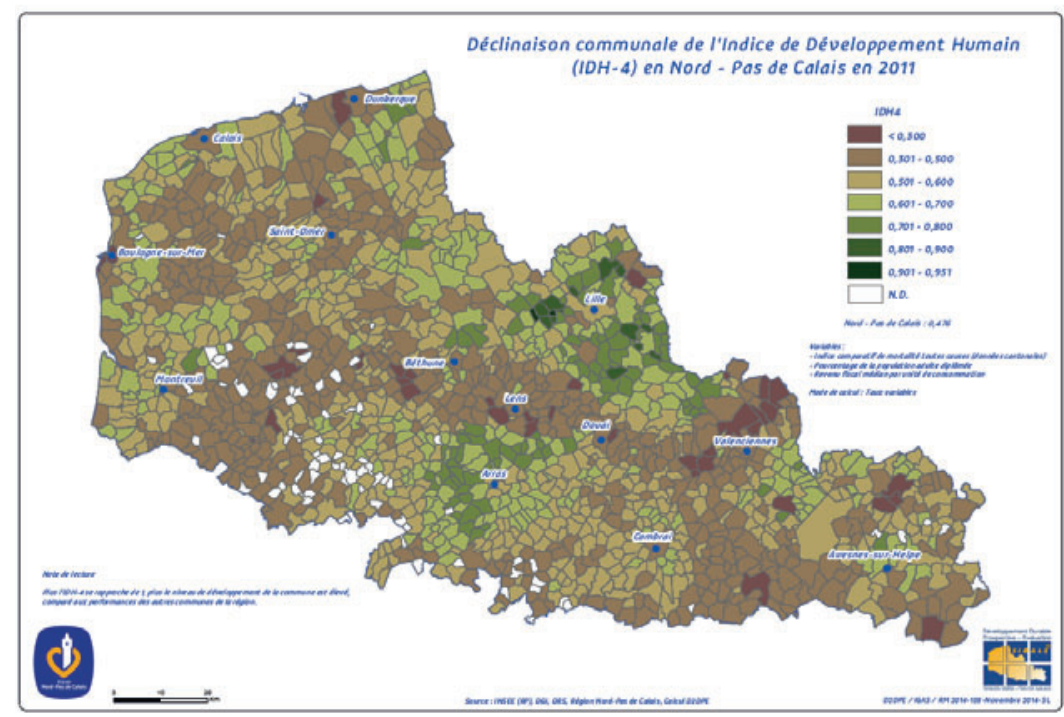

Source : D2DPE, novembre 2014.

De fortes disparités infrarégionales sont mises en évidence : en 2011, l'IDH-4 varie de 0,116 pour la commune d'Avesnes-sur-Helpe à 0,942 pour la commune d'Escobecques dans les Weppes. Avesnes-sur-Helpe $(0,116)$, Roubaix $(0,136)$, Denain $(0,139)$, Condé sur l'Escaut $(0,191)$, Grenay $(0,198)$ et Louvroil $(0,205)$ sont les communes ayant les IDH-4 les plus faibles. Ces communes combinent les difficultés sur les trois dimensions de l'IDH-4. Par exemple, Avesnes-sur-Helpe a un revenu fiscal médian de seulement 11175 euros contre 16797 euros en Nord-Pas-de-Calais, un taux de diplômés inférieur de plus de 9 points à la moyenne régionale et une mortalité supérieure de près de $60 \%$ à celle de la France métropolitaine $(23,9 \%$ pour le Nord-Pas-de-Calais). Parmi les communes ayant les IDH-4 les plus élevés, on retrouve de nombreuses communes situés dans le périurbain lillois ou arrageois. Ces communes ont notamment un revenu fiscal médian supérieur de plus de $60 \%$ à la moyenne régionale. Concernant les principales villes de la région, seules Lille, Arras, Valenciennes et Montreuil-sur-Mer ont un IDH-4 supérieur à la moyenne régionale. Avesnes-sur-Helpe, Boulogne-sur-Mer, Lens, St Omer et Douai ont un indice de développement humain nettement inférieur à cette moyenne régionale (IDH-4 inférieur à 0,4 ). 
Comparé à la seule analyse monétaire de la pauvreté, le calcul de l'IDH-4 permet aussi de mettre en évidence le retard non négligeable de l'ancien bassin minier par rapport à d'autres territoires comme l'Avesnois, pourtant lui aussi en difficulté. Le retard en termes de développement humain de certaines communes de l'ancien bassin minier est amplifié : Sallaumines, Denain, Escaudain, Lourches, Harnes, Noyelles-sous-Lens, .... Ces communes ont un IDH-4 inférieur à 0,4 .

Le découplage développement économique/développement humain est intéressant à souligner sur le territoire de Lens-Hénin dans le bassin minier : avec une reconversion avancée dans le tertiaire, une spécialisation dans des activités porteuses et de vives créations d'unités et d'emplois, ce territoire est dans une dynamique économique proche de la métropole lilloise. Or, ce territoire connaît d'importantes difficultés sociales à la lecture de l'IDH-4 en cumulant les vulnérabilités en termes de niveau de vie, d'éducation et de santé. En dehors de l'ancien bassin minier, plusieurs espaces apparaissent également en difficulté avec un niveau de développement humain inférieur à l'ensemble du Nord-Pas-de-Calais. C'est le cas notamment de plusieurs territoires ruraux qui ont un développement humain en retrait par rapport à la moyenne régionale. C'est en particulier le cas de l'est-Cambrésis dans le Nord, d'une partie des communes des Sept Vallées et de l'arrière pays du Boulonnais dans le Pas-de-Calais. Enfin, le calcul de l'IDH-4 permet de mieux faire apparaître des disparités au sein de certains territoires. Dans l'agglomération lilloise, Roubaix a un IDH-4 inférieur à 0,2 alors que certaines communes du sud de la métropole ont un IDH-4 supérieur à 0,8 . Wattrelos et Tourcoing ont également un IDH-4 inférieur à la moyenne régionale. Ces deux communes ont un revenu fiscal médian et un taux de diplômés dans la population non scolarisée inférieurs à la moyenne régionale.

L'approche multidimensionnelle de l'IDH-4 apporte, à l'échelle des territoires, un regard utile sur la géographie régionale et ses disparités territoriales : au-delà des contrastes observés d'un territoire à l'autre pour les revenus dont disposent les ménages, des spécificités peuvent apparaître quant au niveau global de formation des habitants (dimension éducation) ou aux conditions de santé rencontrées (dimension sanitaire). 
Par exemple, la couverture territoriale en équipements éducatifs (lycées généraux ou professionnels, universités et filières du supérieur) ou en équipements sanitaires (établissements hospitaliers, densité de professions médicales ou maisons de santé) peut moduler le degré de développement humain d'un espace donné. D'autres facteurs sont également à prendre en compte: l'histoire socioéconomique d'un territoire, les pratiques culturelles, le contexte environnemental, ... qui conjointement modulent la situation d'un espace au-delà des seules ressources financières des ménages.

La population jeune est probablement la classe d'âge qui éprouve le plus de difficultés dans ce climat actuel de crise économique. Évaluer son niveau de développement, sa santé socioéconomique, permet de compléter et de nuancer l'analyse précédente en matière de développement humain et d'apporter un élément supplétif de perception du niveau de santé sociale des territoires. L'indice de santé sociale de la jeunesse consiste à cumuler des indices socio-économiques sur les jeunes de la région afin de proposer une vision synthétique de leur situation: les jeunes sont-ils davantage touchés par les difficultés socio-économiques que la population dans son ensemble? L'indice synthétique de la «santé sociale de la jeunesse »a été calculé à partir de 3 indices : le niveau de formation, le manque d'accessibilité à l'emploi, l'entrée dans la vie active par une situation de précarité (AULAB, 2015) ${ }^{6}$.

Dans la majorité des communes de la région Nord-Pas-de-Calais (57\%), les jeunes souffrent d'une situation socio-économique encore plus difficile que la population globale: il s'agit de l'ensemble des communes de l'ancien bassin minier, de la majorité des communes de la métropole lilloise ainsi qu'une partie des communes périurbaines situées entre ces deux ensembles géographiques. C'est également le cas de l'ensemble des pôles urbains régionaux, d'une multitude de communes du littoral et de nombreuses communes rurales (carte 7).

Cet indicateur synthétique, croisé ensuite avec l'IDH-4 apporte une lecture supplémentaire à celui-ci et permet d'analyser le niveau de développement humain des communes de la région Nord-Pas-

6. Ces travaux ont été menés dans le cadre du forum hybride sur les indicateurs de développement animé par la Région Nord-Pas-de-Calais. 
de-Calais au regard de la santé sociale de la jeunesse : un territoire au développement humain élevé est-il nécessairement peuplé par une population jeune également en bonne santé sociale?

Carte 7. Indice de la santé sociale de la jeunesse

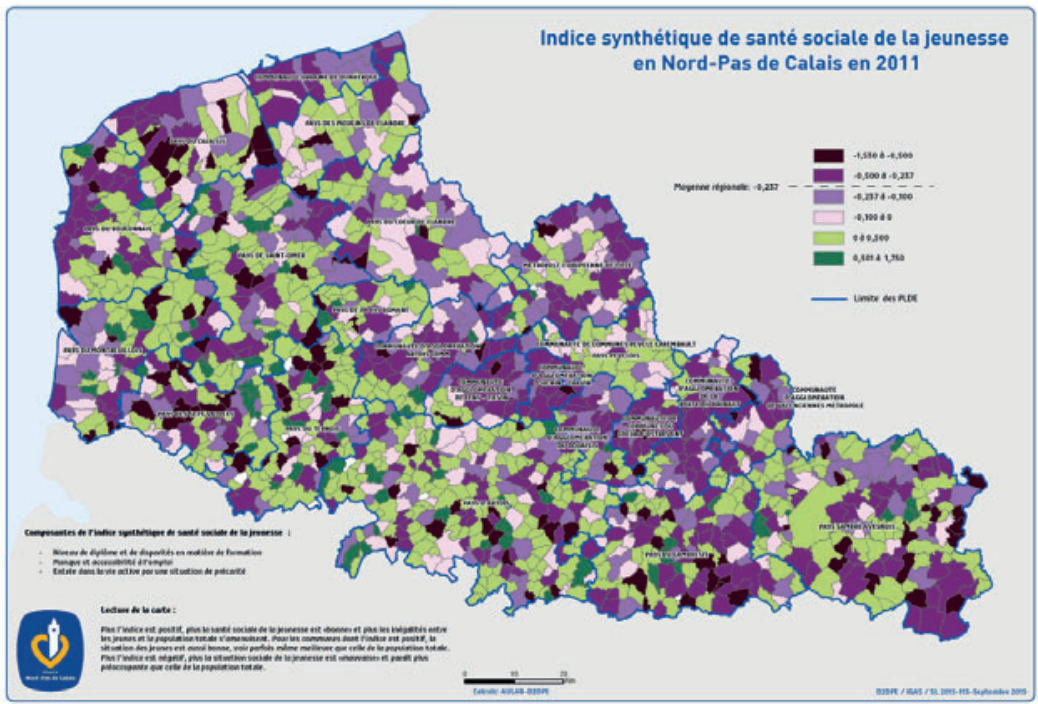

Source : D2DPE, septembre 2015.

Carte 8. Développement humain et indicateur de santé sociale de la jeunesse

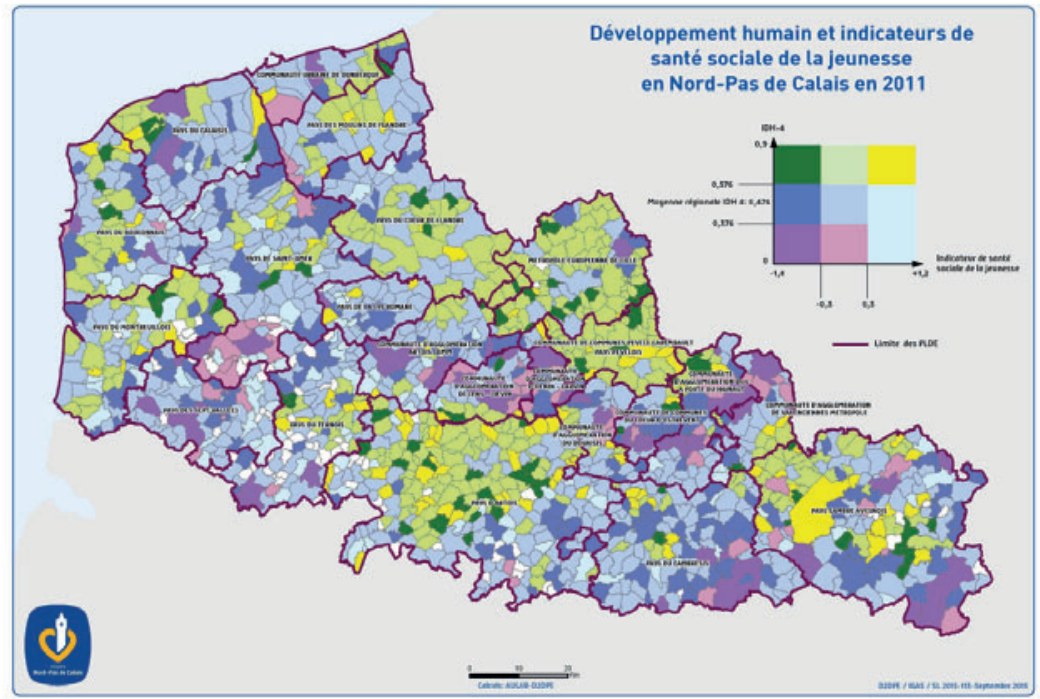

Source : D2DPE, septembre 2015 
Si d'une manière générale, ce nouvel indicateur démontre que de fortes inégalités socio-économiques subsistent entre les jeunes de 15 à 24 ans et le reste de la population, quelques enseignements importants peuvent être soulignés. Le niveau de développement humain de la jeunesse étant très variable d'une commune à une autre, plusieurs grands ensembles semblent se dégager (carte 8).

D'abord, un premier ensemble de communes se distingue où la population cumule un faible niveau de développement humain avec un faible indice de santé sociale de la jeunesse (en violet sur la carte). Autrement dit, les jeunes habitants de ces communes souffrent d'une situation encore plus dégradée que la population totale dont les difficultés socio-économiques sont déjà largement plus marquées qu'ailleurs. Il s'agit principalement des communes de l'ancien bassin minier, auxquelles s'ajoutent les pôles urbains de Boulogne-sur-Mer, de Calais, de Roubaix, ainsi qu'un certain nombre de secteurs plus ruraux. Seules $5 \%$ des communes de la région sont dans cette situation. Cependant, puisqu'elles sont fréquemment de nature urbaine, la population ciblée est en réalité beaucoup plus importante. Ainsi, cet ensemble de communes regroupe en réalité plus de 750000 habitants dont 105000 jeunes de 15 à 24 ans, soit 1 jeune de la région sur 5 .

Ensuite, un deuxième ensemble de communes se distingue assez nettement, rassemblant cette fois les communes périurbaines des principaux pôles urbains régionaux (Lille, Dunkerque, Calais, Boulogne-sur-Mer et Arras). Il s'agit des communes où malgré le niveau de développement humain (IDH-4) très élevé, la population vit en décalage avec ses jeunes dont la santé sociale n'est que moyennement favorable. $22 \%$ des communes sont dans cette situation (vert kaki), soit 790000 habitants dont 98000 jeunes (18\% des 15-24 ans de la région). À noter enfin que sur certaines communes de ce deuxième ensemble, un fossé s'est creusé entre la jeunesse et le reste de la population. En effet, alors que l'IDH-4 y reste élevé, la santé sociale de la jeunesse semble très préoccupante. Il s'agit de communes (vert foncé) où seuls les jeunes de 15 à 24 ans souffrent de difficultés socio-économiques.

Ils pâtissent d'autant plus d'une situation de précarité qu'ils sont en fort décalage avec la population totale dont l'IDH-4 est élevé. 4,5\% des communes sont concernées, soit 100000 habitants dont 12000 jeunes (2,2\% des 15-24 ans). 
En complément de ces approches infra-régionales, croiser la richesse fiscale des territoires et le développement humain de la population conduit à porter un nouveau regard sur les disparités régionales. L'analyse est ici menée à l'échelle de la nouvelle région afin de mettre en perspective les nouveaux enjeux à venir en matière de disparités infrarégionales. La richesse potentielle d'un territoire est couramment utilisée dans des politiques de péréquation nationales, à travers la notion de potentiel financier. Ce potentiel financier est égal au potentiel fiscal, auquel est ajoutée la dotation forfaitaire de la DGF provenant de l'État, perçue par la commune l'année précédente (hors compensation de la suppression de la part salaire de la taxe professionnelle). Ce potentiel financier permet ainsi de prendre en compte l'ensemble des ressources stables d'une collectivité.

Le croisement du potentiel financier avec l'indicateur de développement humain permet de mettre en évidence des territoires doublement pénalisés : population en difficulté sociale au regard du développement humain et faible moyen des collectivités (carte 9).

Pour 41 d'entre elles, le développement humain de la population est très inférieur à la moyenne régionale, tandis que le potentiel financier est très inférieur à la moyenne de la strate démographique de la commune. Elles peuvent être qualifiées de «doublement pauvres ». Il s'agit en particulier de communes du bassin minier comme Avion, Liévin, Montigny en Gohelle, Wingles, Rouvroy, ainsi que des communes aussi différentes que Roubaix ou Montdidier et Creil. À l'inverse, près de 150 communes de la région apparaissent "doublement riches ", bénéficiant d'une population en partie aisée et d'une base fiscale importante. C'est le cas de la proche périphérie de Lille (avec des communes comme Lesquin, Lezennes ou Noyelles-les-Seclin), d'Arras (Duisans) du Grand Beauvaisis ou au sud d'Amiens. Si les petites communes "rurbaines", situées dans les espaces ruraux les plus proches des grandes villes comme Lille, Arras, Amiens ou Senlis, ont également de faibles ressources financières comparées à la moyenne de leur strate démographique, ces dernières accueillent une population plus aisée grâce à leur cadre de vie de qualité, et leur proximité avec de grands centres d'emplois. Dans la région Nord-Pas-de-Calais-Picardie, une centaine de communes correspondent à ce profil. Quelques communes se démarquent par un potentiel financier relativement 
élevé comparé à la moyenne de strate, alors qu'une part importante de leur population combine des difficultés sur les trois dimensions $\mathrm{du}$ développement humain. Sont dans cette situation des communes de taille intermédiaire comme Grande-Synthe, SaintAmand-les-Eaux, ou des communes rurales comme Fruges ou Roye.

Carte 9. Croisement Indicateur de développement humain et potentiel financier

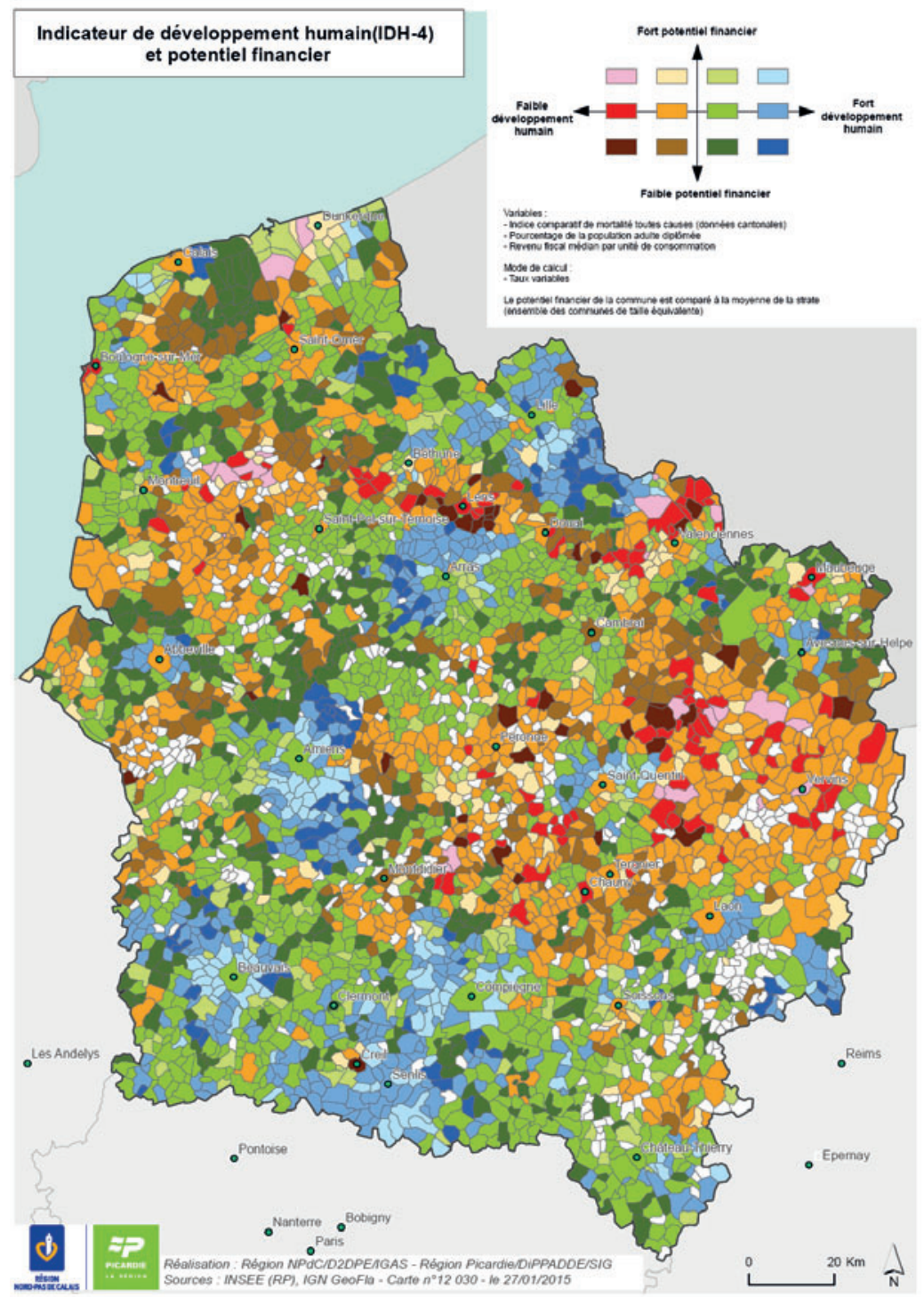




\subsection{Un déterminisme social qui creuse les inégalités}

Une étude économétrique récente publiée dans Economics of Education Review (Ben-Halima, Chusseau et Hellier, 2014) a montré que la mobilité sociale intergénérationnelle a baissé en France. Plus spécifiquement, l'impact du niveau d'étude des parents sur le niveau éducatif des enfants (actifs) est plus fort pour les actifs recensés en 2003 que pour ceux recensés en 1993. L'évolution est donc préoccupante. Elle l'est d'autant plus lorsque l'on compare la France aux autres pays avancés. Un indicateur habituel de la mobilité sociale est l'élasticité intergénérationnelle qui mesure l'impact de l'origine familiale sur les revenus des individus. Plus cette élasticité est forte, plus faible est la mobilité sociale intergénérationnelle. De ce point de vue, on observe une forte hétérogénéité entre économies avancées. Trois pays témoignent d'une faible mobilité sociale intergénérationnelle : les États-Unis, la France et le Royaume Uni, avec des élasticités comprises entre 0,4 et 0,5. À l'opposé se trouvent les pays nordiques, avec des élasticités comprises entre 0,15 et 0,3. Les autres pays se placent entre ces deux groupes. En conséquence, la France a non seulement une faible mobilité sociale intergénérationnelle, mais cette mobilité tend encore à diminuer.

La région Nord-Pas-de-Calais se caractérise par une moindre mobilité intergénérationnelle qu'à l'échelle nationale. En d'autres termes, le changement de catégorie sociale entre un enfant et son père est moins fréquent dans la région qu'à l'échelle nationale : l'écart de mobilité entre région Nord-Pas-de-Calais et France entière est élevé et fortement défavorable à la région $(35,02 \%$ en 1977 contre 44,99 \% en 2003). En outre, la mobilité intergénérationnelle y est plus faiblement ascendante : pour un individu, le passage à une catégorie sociale supérieure à celle de son père est moins fréquent. Parmi 100 personnes nées et résidentes en NordPas-de-Calais, seules 21 connaissent une ascension sociale contre 25 au niveau national (Fleury, 2009).

Cette inertie en termes de catégorie sociale s'observe également en termes de diplômes avec une plus faible probabilité d'obtenir un baccalauréat pour une personne dont le père est sans diplôme (Fleury, 2009). Cette situation, qualifiée de crise de «l'ascenseur social ", a des conséquences sur le rôle que la société confère à l'école avec des problématiques récurrentes d'égalité des chances. 
Elle interroge également la répartition des richesses et le poids des héritages notamment financiers et immobiliers dans les trajectoires individuelles qui contribuent à crisper les relations sociales dans un contexte de montée du sentiment de défiance, envers le futur et envers les autres.

De nombreuses études ont montré que le milieu social influençait la réussite scolaire: ceci est particulièrement vrai pour la France. Une récente étude du ministère de l'Education nationale (MENESR-DEPP, 2015) menée auprès de 35000 élèves de la sixième à la troisième, a comparé les résultats des collégiens à des tests cognitifs portant sur la lecture, les mathématiques, la logique et la mémoire. Le constat est inquiétant: le fossé se creuse entre les élèves issus d'un milieu social aisé et ceux issus d'un milieu social défavorisé. L'enquête PISA 2012 a montré que la France est aujourd'hui le pays de l'OCDE où l'origine sociale des enfants pèse le plus lourd dans les résultats scolaires, cette tendance s'étant accrue ces dix dernières années. Comparativement aux résultats de la précédente enquête PISA 2003, le système éducatif français est plus inégalitaire qu'il ne l'était neuf ans auparavant. On a donc aujourd'hui moins de chance de réussir en France qu'en 2003 lorsque l'on appartient à un milieu défavorisé.

Le système éducatif français se caractérise par le fait qu'il est socialement sélectif. L'origine sociale détermine le type de baccalauréat choisi $(76 \%$ des enfants de cadres et professions intellectuelles supérieures choisissent un bac général alors que $46 \%$ des enfants d'ouvriers s'orientent vers un bac professionnel), ainsi que l'accès à l'enseignement supérieur : toutes formations confondues, $11 \%$ des étudiants sont des enfants d'ouvriers et $30 \%$ sont des enfants de cadres alors que les ouvriers représentent $24 \%$ de la population active contre $15 \%$ pour les cadres. L'écart est encore plus marqué si l'on considère l'accès aux filières élitistes : les enfants de cadres et professions intellectuelles supérieures sont très fortement présents dans les classes préparatoires aux grandes écoles contrairement aux enfants d'ouvriers $(6,3 \%$ des élèves de classes préparatoires sont des enfants d'ouvriers tandis que $49 \%$ ont des parents issus des professions intellectuelles supérieures). De même, $8 \%$ des élèves d'écoles d'ingénieurs sont d'origine ouvrière alors que $56 \%$ ont des parents cadres ou appartenant aux professions intellectuelles supérieures (ministère de l'Éducation nationale, 
données 2012-2013). En France, l'origine sociale est donc déterminante pour le parcours scolaire.

L'impact du milieu social d'origine sur les choix d'éducation est particulièrement important en Nord-Pas-de-Calais. En considérant les inscriptions dans le secondaire général ou technologique dans la région à la rentrée 2014, on constate que $91 \%$ des enfants de cadres sont en LGT contre $49 \%$ des enfants d'ouvriers (tableau 6). Dans le second cycle professionnel, les enfants d'ouvriers et d'employés sont très largement, majoritairement représentés. Les enfants de cadre représentent $25 \%$ des effectifs en LGT et $4 \%$ des effectifs en second cycle professionnel alors que les enfants d'ouvriers représentent $25 \%$ des effectifs en LGT et $45 \%$ des effectifs en second cycle professionnel.

Tableau 6. Inscriptions en secondaire GT ou secondaire PRO selon I'origine socioprofessionnelle des parents (rentrée 2014, Nord-Pas-de-Calais)

En \%

\begin{tabular}{lcc} 
& 2D Cycl. G\&T & 2D Cycl.PRO \\
\hline Cadres & 91 & 9 \\
Professions intermédiaires & 77 & 23 \\
Employés & 65 & 35 \\
Ouvriers & 49 & 51 \\
Ensemble & 63 & 37 \\
\hline
\end{tabular}

Source : Ministère de l'Éducation nationale.

Les inégalités sociales déterminent les inégalités d'éducation, donc les inégalités de revenus. On les retrouve tout au long du cycle de vie avec des conséquences en termes de risque de chômage plus élevé, un niveau de vie à la retraite beaucoup plus faible et un accès à la formation continue également plus limité. De plus, il existe un lien entre inégalités d'éducation, de revenus, d'accès aux soins, d'accès à des logements de bonne qualité, ..., et ces inégalités se transmettent aux générations suivantes.

Lutter contre les inégalités liées au milieu d'origine et permettre aux enfants de milieu modeste de monter dans l'échelle sociale constituent un enjeu majeur pour le Nord-Pas-de-Calais. Dans une région où les inégalités éducatives et culturelles sont fortes et la mobilité intergénérationnelle faible, l'école doit réduire les inégalités liées aux différences familiales avant de sélectionner pour éviter que seuls les enfants issus des milieux cultivés et favorisés 
passent la barrière de la sélection. Il faut donc agir dès les premières années pour compenser les différences sociales et culturelles liées au milieu familial, en favorisant la scolarisation des enfants dès l'âge de deux ans en baisse très sensible au niveau national entre 2000 et 2011. En effet, plus on scolarise tôt les enfants, plus l'activité d'éducation est efficace, particulièrement pour les enfants issus des milieux modestes. En outre, de récents travaux ont montré qu'en présence de fortes inégalités éducatives, il fallait investir massivement dans l'éducation primaire (Chusseau et Hellier, 2011). La mise en œuvre de réformes ciblées sur les élèves en échec scolaire constitue également un levier d'action efficace, tout comme une sélection moins précoce dans le supérieur.

\section{La transition écologique et sociale : réconcilier développement économique, développement humain et développement durable ?}

$\mathrm{Au}$ moment de sa formalisation, la notion de développement durable avait permis de renouveler les objectifs et les pratiques du développement. Marquée par son histoire industrielle, la région Nord-Pas-de-Calais avait été l'une des premières à s'en saisir, elle reste aujourd'hui à la pointe en matière de mise en œuvre de ses principes.

Pourtant, vingt-cinq ans plus tard et malgré le "Grand Tournant » opéré, la situation de la région est toujours aussi délicate tandis que de nouveaux défis se concrétisent (changement climatique, menaces sur la biodiversité, raréfaction des ressources, crises économique et financière, déséquilibres géopolitiques, fragilisation de la cohésion sociale, interrogations sur le rôle des pouvoirs publics, sur la pérennité des fonctionnements démocratiques, etc.) qui rendent les analyses du développement durable en matière de prise en compte du long terme, de croisement des échelles géographiques, d'appréhension systémique des enjeux d'autant plus d'actualité.

Ceci explique la volonté exprimée par la région à travers son Schéma régional d'aménagement et de développement durable du territoire (SRADDT) de s'inscrire dans une deuxième étape du développement durable en Nord-Pas-de-Calais.

Cette nouvelle étape doit s'appuyer sur les acquis de la période précédente tout en mettant en avant trois grands principes : 
- la résilience territoriale qui met l'accent sur la capacité d'un territoire et de ses habitants à rebondir et à s'adapter après un choc, à tirer parti de leurs potentialités pour appréhender les transformations à venir de manière positive ;

- la transition écologique et sociale qui vise à accompagner le changement vers un nouveau modèle de développement plus durable. Pour cela la démarche de la Transformation écologique et sociale constitue un laboratoire d'actions. Elle vise à favoriser la production de biens communs, « eau, air, biodiversité, mais aussi santé au travail, égalité femmeshommes, sécurité économique des travailleurs, préservation de l'environnement, qualité de l'urbanisme, ... C'est-à-dire l'ensemble des biens communs auxquels nous tenons et qui concourent au 'bien-être' durable des populations » (conclusions de la mission d'enquête du SRDE sur les finalités de l'intervention publique dans le domaine de l'économie).

- la mise en ouvre interterritoriale qui place le territoire au cœur des initiatives de développement durable, pour être au plus près des attentes des populations tout en articulant l'ensemble dans un lieu déterminé. Dans ce cadre, la région qui se situe à l'articulation entre le souci du local et la prise en compte des enjeux globaux, a un rôle particulier à jouer.

\section{Références}

Association des régions de France, 2012, "La Révolution des nouveaux indicateurs de développement », Atlas de la santé sociale des régions françaises, 2010, Direction du Développement durable, de la prospective et de l'évaluation, 60 pages, http://www.arf.asso.fr/wp-content/uploads/ 2012/04/rapportfinalARF.pdf

AULAB, 2015, Vers un indicateur de développement humain de la jeunesse, dossier recherche, juin.

Bunel L., 2015, « Commerce extérieur », in Insee Conjoncture Nord-Pasde-Calais, Bilan économique, 5 : 15-19.

Bussière M., G. Callegari, F. Ghironi, G. Sestieri et N. Yamano, 2013, « Estimating Trade Elasticities: Demand Composition and the Trade Collapse of 2008-9 », American Economic Journal: Macroeconomics, 5 : 118-151.

Charles S., L. Cordonnier, T. Dallery et J. Melmiès, 2009, Impact macroéconomique de l'action régionale en matière d'emploi. Construction d'une 
maquette de la macroéconomie régionale, explicitation de la place et de l'impact de l'action publique au travers du multiplicateur keynésien, et appréciation des performances sectorielles, Rapport d'étude pour le Conseil Régional Nord-Pas-de-Calais, 124 pages.

Charles S., L. Cordonnier, T. Dallery et J. Melmiès, 2009, Impact macroéconomique de l'action régionale en matière d'emploi. Construction d'une maquette de la macroéconomie régionale, explicitation de la place et de l'impact de l'action publique au travers du multiplicateur keynésien, et appréciation des performances sectorielles, Rapport d'étude pour le Conseil régional Nord-Pas-de-Calais, 124 pages.

Charles S., L. Cordonnier, T. Dallery et J. Melmiès, 2013, « Le multiplicateur keynésien intra-territorial: le cas de la région Nord-Pas-deCalais », Économie Appliquée, tome LXVI, 2 : 83-113.

Chusseau N., B. Ben-Halima et J. Hellier, 2014, «Skill Premia, and Intergenerational Education Mobility: The French Case », Economics of Education Review, 39 : 50-64, avril.

Chusseau N. et J. Hellier, 2011, «Educational Systems, Intergenerational Mobility and Social Segmentation » (avec J. Hellier), European Journal of Comparative Economics, 8(2) : 203-233.

Creel J., É. Heyer, M. Plane, 2011, « Petit précis de politique budgétaire par tous les temps. Les multiplicateurs budgétaires au cours du cycle », Revue de l'OFCE, 116 : 61-88.

Davezies L., 2009, "L'économie locale 'résidentielle' », Géographie, Économie, Société, 11(1) : 47-53.

Davezies L., 2009, La crise et nos territoires : premiers impacts - Octobre 2010, Rapport préparé pour l'AdCF, la Caisse des Dépôts et l'Institut CDC pour la Recherche.

DIACT, 2009, Dynamiques et développement durable des territoires, Rapport de l'Observatoire des territoires.

Fleury N., 2009, Dynamique des qualifications et mobilité intergénérationnelle dans la région Nord-Pas-de-Calais, Rapport pour la région Nord-Pas-deCalais, document de travail EQUIPPE, Université de Lille 1, France.

Heitz B. et G. Rini, 2006, «Une nouvelle lecture de la contribution du commerce extérieur à la croissance », Trésor-Éco, 6.

INSEE Nord-Pas-de-Calais, 2011 et 2012, « Les espaces du Nord-Pas-deCalais - Trajectoires, enjeux et devenir », Dossiers de Profils, 104, 105, 106, 107.

INSEE Nord-Pas-de-Calais, 2014, « 20 ans de dynamismes économiques en Nord-Pas-de-Calais, création de richesse et mutation du système productif », Page de Profils, 158.

INSEE Nord-Pas-de-Calais, 2009, « Spécificités des territoires du Nord-Pasde-Calais face aux aléas économiques », Page de Profils, 66. 
INSEE Nord-Pas-de-Calais, 2014, « 20 ans de dynamismes économiques en Nord-Pas-de-Calais, création de richesse et mutation du système productif », Page de Profils, 158.

Jany-Catrice F. et G. Marlier, 2013, «Estimer la santé sociale des régions françaises: enjeux économiques, épistémologiques et politiques», Revue d'économie régionale et urbaine, 4 : 647-678.

Jany-Catrice F. et R. Zotti, 2009, « La santé sociale des territoires. Un indicateur de santé sociale pour les régions françaises », Futuribles, 350 : 65-88.

Laurent É., 2013, Vers l'égalité des territoires : Dynamiques, mesures, politiques, Paris, La Documentation française.

MENESR-DEPP, 2015, Note d'information, 25, août.

Région Nord-Pas-de-Calais, 2011, «Économie des territoires du Nord-Pas de Calais, typologie et sensibilité à la crise, quels impacts ? ", Note de la D2DPE, 45.

Région Nord-Pas-de-Calais, 2012, « Un indicateur de Santé Sociale pour les régions françaises : l'ISS, Actualisation pour l'année 2008 », Note de la D2DPE, 49.

Région Nord-Pas-de-Calais, 2015, «Un indicateur de développement humain à l'échelle des territoires du Nord-Pas-de-Calais », Note de la D2DPE, 56. 


\section{ANNEXE 1. Méthodologie de calcul de I'IDH-2}

L'IDH-2 est un indicateur composite formé de 3 sous-indices correspondant aux 3 dimensions retenues: santé, éducation et niveau de vie. Il correspond à une moyenne arithmétique de ces 3 sous-indices.

\begin{tabular}{|c|c|c|c|c|}
\hline $\begin{array}{l}\text { Dimensions du } \\
\text { développement } \\
\text { humain }\end{array}$ & $\begin{array}{l}\text { Variables } \\
\text { choisies }\end{array}$ & $\begin{array}{l}\text { Valeur } \\
\text { minimale }\end{array}$ & $\begin{array}{l}\text { Valeur } \\
\text { maximale }\end{array}$ & Mode de calcul \\
\hline Santé & $\begin{array}{l}\text { Espérance de vie } \\
\text { à la naissance }\end{array}$ & 65 ans & 85 ans & $\begin{array}{l}\text { Taux fixes } \\
\text { Indice de santé = } \\
\text { (espérance de vie - 65)/ } \\
(85-65)]\end{array}$ \\
\hline Éducation & $\begin{array}{l}\text { Pourcentage de la } \\
\text { population âgée de } \\
\text { plus de } 15 \text { ans sortie } \\
\text { du système scolaire } \\
\text { diplômée }\end{array}$ & $50 \%$ & $100 \%$ & $\begin{array}{l}\text { Taux fixes } \\
\text { Indice d'instruction = } \\
\text { (pourcentage de } \\
\text { la population diplômée - } \\
50) /(100-50)\end{array}$ \\
\hline Niveau de vie & $\begin{array}{l}\text { Revenu imposable } \\
\text { médian des ménages } \\
\text { par uc (en } \$ \text { et PPA) }\end{array}$ & 5000 US\$ & 30000 US\$ & $\begin{array}{l}\text { Taux variables } \\
\text { Indice de niveau de vie = } \\
{[\log (\text { revenu médian / uc) - }} \\
\log (5000)] /[\log (30000) \\
-\log (5000)]\end{array}$ \\
\hline
\end{tabular}




\section{ANNEXE 2. Méthodologie de calcul de I'ISS}

\section{Dimensions et variables retenues dans I'ISS}

\begin{tabular}{|c|c|c|c|}
\hline \multirow{2}{*}{ Dimension } & \multirow{2}{*}{$\begin{array}{l}\text { Sous dimension } \\
\text { Consommation }\end{array}$} & \multicolumn{2}{|c|}{ Variables retenues } \\
\hline & & Taux de surendettement & \\
\hline \multirow{3}{*}{ Revenu } & Inégalité et pauvreté & Taux d'ISF & $\begin{array}{l}\text { Montant moyen par ménage } \\
\text { imposable }\end{array}$ \\
\hline & Pauvreté & $\begin{array}{l}\text { Taux de pauvreté monétaire } \\
\text { des moins de } 17 \text { ans }\end{array}$ & \\
\hline & Salaires & $\begin{array}{l}\text { Rapport D9/D1 du niveau de } \\
\text { vie par unité de consommation }\end{array}$ & \\
\hline \multirow{4}{*}{$\begin{array}{l}\text { Travail et } \\
\text { emploi }\end{array}$} & Chômage & Taux de chômage & $\begin{array}{l}\text { Écart taux de chômage entre } \\
\text { femmes et hommes }\end{array}$ \\
\hline & Conditions de travail & $\begin{array}{l}\text { Taux de fréquence des acci- } \\
\text { dents de travail avec arrêt (1) }\end{array}$ & \\
\hline & Précarité & Part de l'emploi précaire & $\begin{array}{l}\text { Taux de travail } \\
\text { à temps partiel }\end{array}$ \\
\hline & $\begin{array}{l}\text { Relations profession- } \\
\text { nelles }\end{array}$ & Taux de conflits du travail (2) & \\
\hline Education & & Taux des actifs sans diplômes & Taux d'accès au baccalauréat \\
\hline Santé & & Espérance de vie à la naissance & \\
\hline Logement & & Taux de recours DALO (3) & \\
\hline Justice & & $\begin{array}{l}\text { Crimes et délits contre les per- } \\
\text { sonnes et les biens pour } \\
100000 \text { habitants }\end{array}$ & \\
\hline Lien social & & $\begin{array}{l}\text { Taux d'adhésion à au moins } \\
\text { une association (4) }\end{array}$ & \\
\hline $\begin{array}{l}\text { Lien } \\
\text { inter- } \\
\text { individuel }\end{array}$ & & $\begin{array}{l}\text { Taux des personnes qui voient } \\
\text { au moins une fois par semaine } \\
\text { leurs amis et leurs voisins (5) }\end{array}$ & \\
\hline
\end{tabular}




\section{ANNEXE 3. Méthode de calcul de I'IDH-4}

L'IDH-4 est un indicateur composite formé de 3 sous-indices correspondant aux 3 dimensions retenues: santé, éducation et niveau de vie. Il correspond à une moyenne arithmétique de ces 3 sous-indices.

\begin{tabular}{|c|c|c|c|c|}
\hline $\begin{array}{l}\text { Dimensions du } \\
\text { développement } \\
\text { humain }\end{array}$ & $\begin{array}{l}\text { Variables } \\
\text { choisies }\end{array}$ & $\begin{array}{l}\text { Valeur } \\
\text { minimale }\end{array}$ & $\begin{array}{c}\text { Valeur } \\
\text { maximale }\end{array}$ & Mode de calcul \\
\hline Santé & $\begin{array}{l}\text { Complément de } \\
\text { l'indice Comparatif } \\
\text { de mortalité } \\
\text { (toutes causes) }\end{array}$ & $\begin{array}{c}\text { Min } \\
\text { observée }\end{array}$ & $\begin{array}{c}\text { Max } \\
\text { observée }\end{array}$ & $\begin{array}{l}\text { Taux variables } \\
\text { Indice de santé }= \\
1-[(I C M-\min ) \\
/(\max -\min )]\end{array}$ \\
\hline Éducation & $\begin{array}{l}\text { Pourcentage de la } \\
\text { population âgée de plus } \\
\text { de } 15 \text { ans sortie du sys- } \\
\text { tème scolaire diplômée }\end{array}$ & $\begin{array}{c}\text { Min } \\
\text { observée }\end{array}$ & $\begin{array}{c}\text { Max } \\
\text { observée }\end{array}$ & $\begin{array}{l}\text { Taux variables } \\
\text { Indice d'instruction = } \\
\text { (pourcentage de la popu- } \\
\text { lation diplômée }-\mathrm{min}) / \\
(\max -\min )\end{array}$ \\
\hline Niveau de vie & $\begin{array}{l}\text { Revenu imposable } \\
\text { médian des ménages } \\
\text { par uc (en } \$ \text { et PPA) }\end{array}$ & $\begin{array}{c}\text { Min } \\
\text { observée }\end{array}$ & $\begin{array}{c}\text { Max } \\
\text { observée }\end{array}$ & $\begin{array}{l}\text { Taux variables } \\
\text { Indice de niveau de vie }=[ \\
\log (\text { revenu médian } / \text { uc }) \\
-\log (\min )] /[\log (\max )- \\
\log (\min )]\end{array}$ \\
\hline
\end{tabular}


